\title{
AN AMALGAMATION OF THE BANACH SPACES ASSOCIATED WITH JAMES AND SCHREIER, PART I: BANACH-SPACE STRUCTURE
}

\author{
ALISTAIR BIRD and NIELS JAKOB LAUSTSEN \\ Department of Mathematics and Statistics, Lancaster University \\ Lancaster LA1 4YF, England \\ E-mail:a.bird@lancaster.ac.uk,n.laustsen@lancaster.ac.uk
}

\begin{abstract}
We create a new family of Banach spaces, the James-Schreier spaces, by amalgamating two important classical Banach spaces: James' quasi-reflexive Banach space on the one hand and Schreier's Banach space giving a counterexample to the Banach-Saks property on the other. We then investigate the properties of these James-Schreier spaces, paying particular attention to how key properties of their 'ancestors' (that is, the James space and the Schreier space) are expressed in them. Our main results include that each James-Schreier space is $c_{0}$-saturated and that no James-Schreier space embeds in a Banach space with an unconditional basis.
\end{abstract}

1. Introduction. The purpose of this paper is to introduce a new family of Banach spaces which we call the James-Schreier spaces because they arise by amalgamating the definitions of the quasi-reflexive James spaces with the Schreier space. The original motivation behind these spaces was to produce a new example of a Banach sequence algebra with a bounded approximate identity, in analogy with Andrew and Green's study of the James space as a Banach algebra [3]. This idea turned out to be successful, as essentially all results about the James space as a Banach algebra carry over to our new spaces; see [9] for details.

Having thus reached our initial goal, we soon realized that a serious problem was lurking in the background, namely: how can we distinguish the James-Schreier spaces from the James spaces? Obviously, if they were isomorphic, our findings would be of no interest. In order to resolve this problem, we turned to Banach-space properties and, as we shall see, at that level differences abound; this is the main theme of the present paper.

2010 Mathematics Subject Classification: Primary 46B45; Secondary 46B03, 47B37.

Key words and phrases: Banach space, James space, Schreier space, shrinking basis, shift operator, $c_{0}$-saturation, Pełczyński's property $(\mathrm{u})$.

The paper is in final form and no version of it will be published elsewhere. 
We begin with surveys of the James spaces (Section 2) and the Schreier space (Section 3), where we introduce notation and explain ideas and properties which we shall subsequently pursue in the case of the James-Schreier spaces. In fact, as a spin-off of our investigation, we generalize the concept of a Schreier space from just one Schreier space (corresponding to the $\ell_{1}$-norm) to a whole family, one for each $p \in[1, \infty)$ (corresponding to the $\ell_{p}$-norms). The basic theory of these spaces is developed in Section 3 , our most important findings are: (i) the standard unit vector basis is a shrinking, 1-unconditional basis for each Schreier space; (ii) those right-shifts which define bounded operators on the Schreier spaces can be characterized (see Corollary 3.17) (ii) for details). The former of these results leads to an explicit description of the biduals of the Schreier spaces, while the latter implies that each Schreier space is isomorphic to its Cartesian square.

Section 4 contains the definition of the James-Schreier spaces, one for each $p \in[1, \infty)$, together with an exposition of their basic theory. Results include: (i) the standard basis is a monotone basis, shrinking for $p>1$, but not unconditional (it will follow from results in Section 6 that no basis is); (ii) each James-Schreier space contains a complemented copy of the corresponding Schreier space; (iii) the standard right shift defines a bounded operator on the James-Schreier spaces only in the trivial cases, but a suitably modified version of it turns out to be bounded under conditions similar to those found for the Schreier spaces (see Propositions 4.18 ii) and 4.20.

Sections 5 and 6 contain our two main results: the James-Schreier spaces are $c_{0}$-saturated (which means that each of their closed, infinite-dimensional subspaces contains a copy of $\left.c_{0}\right)$, and they do not have Pełczyński's property $(\mathrm{u})$, so in particular they do not embed in a Banach space with an unconditional basis.

As a consequence of these results, we can complete the 'comparison theory' of our spaces: firstly, the James spaces are totally incomparable with both the Schreier and the James-Schreier spaces (which means that they have no closed, infinite-dimensional subspaces in common), and secondly, no James-Schreier space embeds in a Schreier space, whereas each Schreier space embeds complementedly in a James-Schreier space, as already mentioned in (ii) above.

We conclude this introduction with some general conventions on notation and terminology. Throughout, $\mathbb{K}$ denotes the scalar field; either $\mathbb{K}=\mathbb{R}$ or $\mathbb{K}=\mathbb{C}$.

By an operator we understand a linear mapping between vector spaces. We write $I_{X}$ for the identity operator on a vector space $X$. A functional is a linear mapping from a vector space to its scalar field. For a functional $f$ on a vector space $X$ and a vector $x \in X$, we usually write $\langle x, f\rangle$ instead of $f(x)$.

Let $X$ be a normed space. For a subset $M$ of $X$, span $M$ denotes the linear span of $M$ in $X$, while $\overline{\operatorname{span}} M$ is its closure. We write $X^{\prime}$ for the (continuous) dual of $X$, that is, $X^{\prime}$ consists of the bounded functionals on $X$. The canonical embedding of $X$ into its bidual $X^{\prime \prime}$ is denoted by $\kappa_{X}$, or just $\kappa$ if reference to $X$ is unnecessary. We say that two normed spaces $X$ and $Y$ are isomorphic, written $X \cong Y$, if there is a linear homeomorphism from $X$ onto $Y$.

By a basis for a Banach space $X$, we shall always understand a Schauder basis, that is, a sequence $\left(b_{n}\right)_{n \in \mathbb{N}}$ in $X$ such that, for each $x \in X$, there is a unique sequence $\left(\alpha_{n}\right)_{n \in \mathbb{N}}$ 
of scalars such that the series $\sum_{n=1}^{\infty} \alpha_{n} b_{n}$ is norm-convergent with sum $x$. If this series converges unconditionally for each $x \in X$, then the basis $\left(b_{n}\right)_{n \in \mathbb{N}}$ is unconditional. A sequence $\left(b_{n}\right)_{n \in \mathbb{N}}$ in a Banach space is a basic sequence if $\left(b_{n}\right)_{n \in \mathbb{N}}$ is a basis for its closed linear span, $\overline{\operatorname{span}}\left\{b_{n}: n \in \mathbb{N}\right\}$.

For $m \in \mathbb{N}$, the $m^{\text {th }}$ biorthogonal functional $b_{m}^{\prime}$ associated with a basis $\left(b_{n}\right)_{n \in \mathbb{N}}$ for a Banach space $X$ is given by $\left\langle x, b_{m}^{\prime}\right\rangle:=\alpha_{m}$ for each $x=\sum_{n=1}^{\infty} \alpha_{n} b_{n} \in X$. This is a bounded functional on $X$, and $\left(b_{m}^{\prime}\right)_{m \in \mathbb{N}}$ is a basic sequence in $X^{\prime}$. If $\left(b_{m}^{\prime}\right)_{m \in \mathbb{N}}$ is a basis for $X^{\prime}$ (that is, if $\overline{\operatorname{span}}\left\{b_{m}^{\prime}: m \in \mathbb{N}\right\}=X^{\prime}$ ), then the basis $\left(b_{n}\right)_{n \in \mathbb{N}}$ is shrinking.

We denote by $\mathbb{K}^{\mathbb{N}}$ the vector space of all sequences over $\mathbb{K} ; c_{00}$ is the subspace of $\mathbb{K}^{\mathbb{N}}$ of finitely supported sequences. The vectors

$$
e_{n}:=(0,0, \ldots, 0, \underset{\text { pos. } n}{1}, 0,0, \ldots) \quad(n \in \mathbb{N})
$$

form a Hamel basis for $c_{00}$, called the standard unit vector basis. For $m \in \mathbb{N}$, the $m^{\text {th }}$ coordinate functional is given by

$$
f_{m}:\left(\alpha_{n}\right)_{n \in \mathbb{N}} \mapsto \alpha_{m}, \quad \mathbb{K}^{\mathbb{N}} \rightarrow \mathbb{K},
$$

and for a set $A$ (usually a subset of $\mathbb{N}$ ), the natural projection associated with $A$ is the operator $P_{A}: \mathbb{K}^{\mathbb{N}} \rightarrow \mathbb{K}^{\mathbb{N}}$ whose coordinates are given by

$$
\left\langle P_{A} x, f_{m}\right\rangle:=\left\{\begin{array}{ll}
\left\langle x, f_{m}\right\rangle & \text { if } m \in A \\
0 & \text { otherwise }
\end{array} \quad\left(m \in \mathbb{N}, x \in \mathbb{K}^{\mathbb{N}}\right) .\right.
$$

This is clearly an idempotent operator. In the special case where $A=\{1,2, \ldots, m\}$ for some $m \in \mathbb{N}$, we write $P_{m}$ instead of $P_{A}$, that is,

$$
P_{m}:\left(\alpha_{n}\right)_{n \in \mathbb{N}} \mapsto\left(\alpha_{1}, \ldots, \alpha_{m}, 0,0, \ldots\right), \quad \mathbb{K}^{\mathbb{N}} \rightarrow c_{00} ;
$$

we call $P_{m}$ the $m^{\text {th }}$ natural projection.

For a set $A$, we define $\chi_{A} \in \mathbb{K}^{\mathbb{N}}$ by

$$
\left\langle\chi_{A}, f_{m}\right\rangle=\left\{\begin{array}{ll}
1 & \text { if } m \in A \\
0 & \text { otherwise }
\end{array} \quad(m \in \mathbb{N}) ;\right.
$$

the most important cases are

$$
\chi_{[n, n+k]}=\sum_{j=n}^{n+k} e_{j} \in c_{00} \quad(n, k \in \mathbb{N}) \quad \text { and } \quad \chi_{\mathbb{N}}=(1,1, \ldots, 1,1, \ldots) .
$$

Given a set $B$, we write $A \Subset B$ to indicate that $A$ is a finite subset of $B$. In the case where $B$ is totally ordered (typically $B=\mathbb{N}$ ), we write $A=\left\{n_{1}<n_{2}<\cdots<n_{k}\right\}$ to signify that $\left\{n_{1}, n_{2}, \ldots, n_{k}\right\}$ is the increasing ordering of $A$.

We denote by card $A$ the cardinality of a set $A$. We shall not be concerned with the subtleties of infinite cardinalities because we shall only consider cardinalities of subsets of $\mathbb{N}$; hence we think of card $A$ as belonging to $\{0,1,2, \ldots, \infty\}$.

2. James' quasi-reflexive Banach spaces. This section contains a brief survey of the James spaces and their most important properties, with special emphasis on the Banach-algebraic aspects; no proofs will be given. However, at the end we shall outline 
James' original construction because our subsequent work will involve key components of it. An elementary introduction to the James spaces is given in [30, while a much more comprehensive account can be found in [18; both of these references contain full proofs.

The original James space $J_{2}$ was introduced by James in [22]. His main result states that this Banach space is isomorphic to its bidual and quasi-reflexive (meaning that the canonical image of $J_{2}$ in its bidual has codimension 1). This resolved two major open problems at the time:

(i) A Banach space with separable bidual need not be reflexive.

(ii) A separable Banach space which is isomorphic to its bidual need not be reflexive.

In a sequel [23], James defined an equivalent norm on $J_{2}$ with respect to which the space even becomes isometrically isomorphic to its bidual.

Subsequently, many other interesting properties of the James space have been added to James' list; we mention some of the most important here:

(iii) Bessaga and Pełczyński [8] observed that the quasi-reflexivity of $J_{2}$ implies that $J_{2} \nsucceq J_{2} \oplus J_{2}$, making $J_{2}$ the first known example of an infinite-dimensional Banach space which is not isomorphic to its Cartesian square.

(iv) Herman and Whitley [21] proved that $J_{2}$ is $\ell_{2}$-saturated, that is, every closed, infinite-dimensional subspace of $J_{2}$ contains a subspace $X$ which is isomorphic to $\ell_{2}$; Casazza, Lin, and Lohman [14] subsequently showed that it is always possible to choose such an $X$ with the additional property that $X$ is complemented in $J_{2}$.

(v) Edelstein and Mityagin [17] calculated the homotopy type of the group of invertible operators on $J_{2}$. As part of this calculation, they noted that the quasireflexivity of $J_{2}$ has the easy, but algebraically very important, consequence that the ideal $\mathscr{W}\left(J_{2}\right)$ of weakly compact operators has codimension 1 in the Banach algebra $\mathscr{B}\left(J_{2}\right)$ of bounded operators on $J_{2}$, so that there is a character on $\mathscr{B}\left(J_{2}\right)$. No such examples were previously known. Laustsen [25, 26] has subsequently shown that $\mathscr{W}\left(J_{2}\right)$ is the unique maximal ideal in $\mathscr{B}\left(J_{2}\right)$, while the scalar multiples of the character are the only traces on $\mathscr{B}\left(J_{2}\right)$.

(vi) Giesy and James [20] have shown that $c_{0}$ is finitely representable in $J_{2}$, that is, for each $\varepsilon>0$ and each $n \in \mathbb{N}$, there is an operator $T: \ell_{\infty}^{n} \rightarrow J_{2}$ such that

$$
(1-\varepsilon)\|x\|_{\ell_{\infty}^{n}} \leqslant\|T x\|_{J_{2}} \leqslant(1+\varepsilon)\|x\|_{\ell_{\infty}^{n}} \quad\left(x \in \ell_{\infty}^{n}\right) .
$$

Consequently, $J_{2}$ does not have finite coptype. In contrast, Pisier [33] has proved that the dual $J_{2}^{\prime}$ has cotype 2 and, moreover, that $J_{2}$ has the Gordon-Lewis property (which means that every 1-summing operator from $J_{2}$ to an arbitrary Banach space factors through $L_{1}$ ).

(vii) Casazza [13] has shown that $J_{2}$ is primary, that is, $X \cong J_{2}$ or $Y \cong J_{2}$ whenever $X$ and $Y$ are closed, complementary subspaces of $J_{2}$.

(viii) Andrew and Green [3] have shown that $J_{2}$ is a Banach algebra with respect to the pointwise product. Further, this Banach algebra is Arens regular, and its multiplier algebra can be identified with $J_{2}^{\prime \prime}$ with the Arens product.

(ix) Loy and Willis [29] have constructed a bounded right approximate identity in the ideal $\mathscr{W}\left(J_{2}\right)$ of weakly compact operators on $J_{2}$. This allowed them to deduce that 
derivations from $\mathscr{B}\left(J_{2}\right)$ are automatically continuous. Willis [35] has subsequently generalized this result by showing that homomorphisms from $\mathscr{B}\left(J_{2}\right)$ are automatically continuous.

(x) Odell and Tylli [31] completed Loy and Willis' work on bounded approximate identities in $\mathscr{W}\left(J_{2}\right)$ by showing that $\mathscr{W}\left(J_{2}\right)$ also has a bounded left approximate identity and hence a bounded two-sided approximate identity.

(xi) Laustsen [24] has calculated the $K$-groups of the Banach algebra $\mathscr{B}\left(J_{2}\right)$.

(xii) Blanco [11 has proved that the ideal $\mathscr{K}\left(J_{2}\right)$ of compact operators on $J_{2}$ is weakly amenable. It is an open problem if $\mathscr{K}\left(J_{2}\right)$ is amenable; Blanco and Grønbæk [12] have recently shown that $\mathscr{K}\left(J_{2} \oplus J_{2}^{\prime}\right)$ fails to be amenable.

We shall now take a closer look at the formal definition of the James space and the strategy which James followed when he proved that it is quasi-reflexive.

2.1. The James spaces. Let $1 \leqslant p<\infty$. For $x=\left(\alpha_{n}\right)_{n \in \mathbb{N}} \in \mathbb{K}^{\mathbb{N}}$ and $A \Subset \mathbb{N}$, define

$$
\nu_{p}(x, A):= \begin{cases}0 & \text { when card } A \leqslant 1 ; \\ \left(\sum_{j=1}^{k}\left|\alpha_{n_{j}}-\alpha_{n_{j+1}}\right|^{p}\right)^{\frac{1}{p}} & \text { when } A=\left\{n_{1}<\cdots<n_{k+1}\right\} \text { for some } k \in \mathbb{N} .\end{cases}
$$

Using Minkowski's inequality, one can easily check that $\nu_{p}(\cdot, A)$ is a seminorm on $\mathbb{K}^{\mathbb{N}}$ for each $A \Subset \mathbb{N}$, and

$$
\begin{aligned}
\|x\|_{J_{p}} & :=\sup \left\{\nu_{p}(x, A): A \Subset \mathbb{N}\right\} \\
& =\sup \left\{\left(\sum_{j=1}^{k}\left|\alpha_{n_{j}}-\alpha_{n_{j+1}}\right|^{p}\right)^{\frac{1}{p}}: k, n_{1}, \ldots, n_{k+1} \in \mathbb{N}, n_{1}<n_{2}<\cdots<n_{k+1}\right\}
\end{aligned}
$$

defines a complete norm on the subspace $J_{p}:=\left\{x \in c_{0}:\|x\|_{J_{p}}<\infty\right\}$ of $\mathbb{K}^{\mathbb{N}}$. The Banach space $\left(J_{p},\|\cdot\|_{J_{p}}\right)$ is called the $p^{\text {th }}$ James space.

REMARK 2.2. James' original definition corresponds to the case $p=2$ above. Edelstein and Mityagin [17] appear to have been the first to observe that it can be generalized to arbitrary $p \geqslant 1$ and, moreover, that James' proof of the quasi-reflexivity of $J_{2}$ carries over directly to $J_{p}$ for each $p>1$. The proof does not, however, work for $p=1$ because $J_{1}$ is isometrically isomorphic to $\ell_{1}$ via the mapping $\left(\alpha_{n}\right)_{n \in \mathbb{N}} \mapsto\left(\alpha_{n}-\alpha_{n+1}\right)_{n \in \mathbb{N}}, J_{1} \rightarrow \ell_{1}$.

Most of the results (iii)-xii) listed above have generalizations to $J_{p}$ for $p>1$. We state the following generalization of (iv) explicitly for later reference.

Proposition 2.3. Let $p>1$. Then every closed, infinite-dimensional subspace of $J_{p}$ contains a subspace which is isomorphic to $\ell_{p}$ and complemented in $J_{p}$.

The key element in James' proof [22] that $J_{2}$ is quasi-reflexive is the following explicit description of the bidual of a Banach space with a shrinking basis. We follow Megginson's presentation [30, Section 4.5]. 
2.4. The James representation of the bidual of a Banach space with a shrinking basis. For a Banach space $X$ with a fixed basis $\left(b_{n}\right)_{n \in \mathbb{N}}$, define

$$
\|x\|_{\operatorname{bip}(X)}:=\sup \left\{\left\|\sum_{n=1}^{m} \alpha_{n} b_{n}\right\|_{X}: m \in \mathbb{N}\right\} \quad\left(x=\left(\alpha_{n}\right)_{n \in \mathbb{N}} \in \mathbb{K}^{\mathbb{N}}\right) .
$$

Then

$$
\operatorname{bip}(X):=\left\{x \in \mathbb{K}^{\mathbb{N}}:\|x\|_{\operatorname{bip}(X)}<\infty\right\}
$$

is a subspace of $\mathbb{K}^{\mathbb{N}}$, and $\|\cdot\|_{\operatorname{bip}(X)}$ is a complete norm on it.

Now suppose that the basis $\left(b_{n}\right)_{n \in \mathbb{N}}$ is shrinking. Then the operator $\Upsilon: X^{\prime \prime} \rightarrow \operatorname{bip}(X)$ given by $\Upsilon(F):=\left(\left\langle b_{n}^{\prime}, F\right\rangle\right)_{n \in \mathbb{N}}$ for each $F \in X^{\prime \prime}$ is an isomorphism which makes the diagram

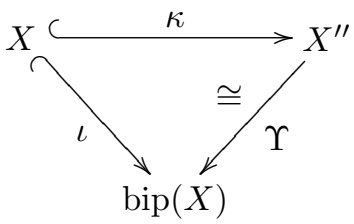

commutative, where $\iota$ is the operator $x \mapsto\left(\left\langle x, b_{n}^{\prime}\right\rangle\right)_{n \in \mathbb{N}}$, while $\kappa$ is the canonical embedding. The isomorphism $\Upsilon$ is isometric if the basis $\left(b_{n}\right)_{n \in \mathbb{N}}$ is monotone.

REMARK 2.5. There is no standard notation for the Banach space $\operatorname{bip}(X)$ considered in 2.4. we have chosen the letters bip for 'bounded initial projections'.

2.6. The quasi-reflexivity of the James spaces. The starting point of the proof that $J_{p}$ is quasi-reflexive for each $p>1$ is the observation that $\left(e_{n}\right)_{n \in \mathbb{N}}$ is a monotone, shrinking basis for $J_{p}$, so that the results of $\$ 2.4$ apply. One then checks that $J_{p}$ and $\mathbb{K} \chi_{\mathbb{N}}$ are closed, complementary subspaces of $\operatorname{bip}\left(J_{p}\right)$, and we therefore have a commutative diagram

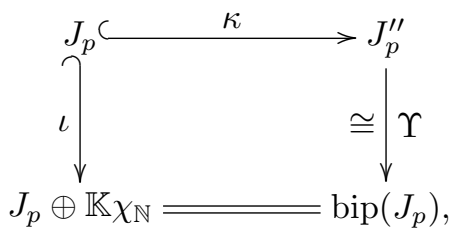

where $\iota$ is the natural inclusion, $\kappa$ is the canonical embedding, and $\Upsilon$ is the isometric isomorphism from \$2.4. This shows immediately that $\kappa\left(J_{p}\right)$ has codimension 1 in $J_{p}^{\prime \prime}$ and, moreover, if we identify $J_{p}^{\prime \prime}$ with $\operatorname{bip}\left(J_{p}\right)$ via $\Upsilon$, then $\kappa=\iota$, that is, the canonical embedding of $J_{p}$ into its bidual becomes the natural inclusion of $J_{p}$ into $J_{p} \oplus \mathbb{K} \chi_{\mathbb{N}}$.

3. The Schreier spaces. Having proved that, for $1<p<\infty$, each weakly convergent sequence $\left(x_{n}\right)_{n \in \mathbb{N}}$ in $L_{p}[0,1]$ has a subsequence $\left(x_{n_{j}}\right)_{j \in \mathbb{N}}$ whose associated sequence of arithmetic means, $\left(\frac{1}{m} \sum_{j=1}^{m} x_{n_{j}}\right)_{m \in \mathbb{N}}$, is norm-convergent, Banach and Saks [5] went on to ask if a similar result would be true in $C[0,1]$. Schreier [34 answered this question in the negative; his counterexample was based on the following notion.

Definition 3.1. A subset $A$ of $\mathbb{N}$ is admissible if $1 \leqslant \operatorname{card} A \leqslant \min A$. 
Thus, an admissible set is non-empty and finite, and for $A=\left\{n_{1}<\cdots<n_{k}\right\} \Subset \mathbb{N}$, we have

$$
A \text { is admissible } \Leftrightarrow k \leqslant n_{1} \text {. }
$$

Each non-empty subset of an admissible set is itself admissible; we shall use this simple observation frequently.

3.2. The unrestricted Schreier spaces. Let $1 \leqslant p<\infty$. For $x=\left(\alpha_{n}\right)_{n \in \mathbb{N}} \in \mathbb{K}^{\mathbb{N}}$ and $A \subseteq \mathbb{N}$, define $\mu_{p}(x, A):=\left(\sum_{n \in A}\left|\alpha_{n}\right|^{p}\right)^{\frac{1}{p}}$. (Note: the sum over an empty index set is zero by convention.) Minkowski's inequality implies that $\mu_{p}(\cdot, A)$ is a seminorm on $\mathbb{K}^{\mathbb{N}}$ for each $A \Subset \mathbb{N}$, and

$$
\begin{aligned}
\|x\|_{Z_{p}} & :=\sup \left\{\mu_{p}(x, A): A \subseteq \mathbb{N} \text { is admissible }\right\} \\
& =\sup \left\{\left(\sum_{j=1}^{k}\left|\alpha_{n_{j}}\right|^{p}\right)^{\frac{1}{p}}: k, n_{1}, \ldots, n_{k} \in \mathbb{N}, k \leqslant n_{1}<n_{2}<\cdots<n_{k}\right\}
\end{aligned}
$$

then defines a norm on the subspace $Z_{p}:=\left\{x \in \mathbb{K}^{\mathbb{N}}:\|x\|_{Z_{p}}<\infty\right\}$ of $\mathbb{K}^{\mathbb{N}}$. We call $\|\cdot\|_{Z_{p}}$ the $p^{\text {th }}$ Schreier norm and $Z_{p}$ the $p^{\text {th }}$ unrestricted Schreier space.

The following lemma records some basic facts about this space; we omit its easy proof.

Lemma 3.3. Let $p \geqslant 1$. Then:

(i) for each $m \in \mathbb{N}$, the restriction to $Z_{p}$ of the $m^{\text {th }}$ coordinate functional $f_{m}$ given by 1.2 is bounded with norm 1 ;

(ii) $\left\langle x, f_{m}\right\rangle \rightarrow 0$ as $m \rightarrow \infty$ for each $x \in Z_{p}$;

(iii) for each $A \subseteq \mathbb{N}, Z_{p}$ is an invariant subspace for the projection $P_{A}$ given by (1.3), and the restriction to $Z_{p}$ of $P_{A}$ is bounded with norm 1 (except for $A=\emptyset$, in which case $\left.P_{A}=0\right)$;

(iv) $Z_{p}$ is a Banach space with respect to the coordinatewise defined operations inherited from $\mathbb{K}^{\mathbb{N}}$ and the norm $\|\cdot\|_{Z_{p}}$.

3.4. The unconditional multiplier constant. Suppose that $\left(b_{n}\right)_{n \in \mathbb{N}}$ is an unconditional basic sequence in a Banach space, and let $\left(\alpha_{n}\right)_{n \in \mathbb{N}}$ be a scalar sequence such that the series $\sum_{n=1}^{\infty} \alpha_{n} b_{n}$ converges. Then, for each scalar sequence $\left(\beta_{n}\right)_{n \in \mathbb{N}}$ with $\left|\beta_{n}\right| \leqslant\left|\alpha_{n}\right|$ for each $n \in \mathbb{N}$, the series $\sum_{n=1}^{\infty} \beta_{n} b_{n}$ converges, and there is a constant $K \geqslant 1$ (independent of $\left(\alpha_{n}\right)_{n \in \mathbb{N}}$ and $\left.\left(\beta_{n}\right)_{n \in \mathbb{N}}\right)$ such that $\left\|\sum_{n=1}^{\infty} \beta_{n} b_{n}\right\| \leqslant K\left\|\sum_{n=1}^{\infty} \alpha_{n} b_{n}\right\|$. The smallest such constant $K$ is the unconditional multiplier constant of $\left(b_{n}\right)_{n \in \mathbb{N}}$. If $K=1$ (the smallest possible), then $\left(b_{n}\right)_{n \in \mathbb{N}}$ is 1-unconditional (or monotone unconditional).

Proposition 3.5. For each $p \geqslant 1$, the sequence $\left(e_{n}\right)_{n \in \mathbb{N}}$ given by (1.1) is a normalized, 1-unconditional basic sequence in $Z_{p}$.

Proof. Clearly we have $\left\|e_{n}\right\|_{Z_{p}}=1$ for each $n \in \mathbb{N}$. Suppose that $x=\sum_{n=1}^{\infty} \alpha_{n} e_{n}$ and $y=\sum_{n=1}^{\infty} \beta_{n} e_{n}$ are vectors in $c_{00}$ with $\left|\beta_{n}\right| \leqslant\left|\alpha_{n}\right|$ for each $n \in \mathbb{N}$. This implies that $\mu_{p}(y, A)^{p} \leqslant \mu_{p}(x, A)^{p}$ for each subset $A$ of $\mathbb{N}$, and consequently $\|y\|_{Z_{p}} \leqslant\|x\|_{Z_{p}}$. The conclusion now follows from the 1-unconditional counterpart of Banach's fundamental characterization of basic sequences (analogous to [30, Corollary 4.2.33], for instance). 
3.6. The restricted Schreier spaces. Lemma 3.3 iv and Proposition 3.5 imply that

$$
S_{p}:=\overline{\operatorname{span}}\left\{e_{n}: n \in \mathbb{N}\right\} \subseteq Z_{p}
$$

is a Banach space with a normalized, 1 -unconditional basis $\left(e_{n}\right)_{n \in \mathbb{N}}$ for each $p \geqslant 1$. We call $S_{p}$ the $p^{\text {th }}$ (restricted) Schreier space and refer to $\left(e_{n}\right)_{n \in \mathbb{N}}$ as its standard unit vector basis. More precisely, we usually call $S_{p}$ just the $p^{\text {th }}$ Schreier space, inserting the adjective 'restricted' only if confusion with the unrestricted counterpart $Z_{p}$ seems likely. We write $\|\cdot\|_{S_{p}}$ for the restriction to $S_{p}$ of the norm $\|\cdot\|_{Z_{p}}$.

Note that the $m^{\text {th }}$ biorthogonal functional associated with the standard unit vector basis $\left(e_{n}\right)_{n \in \mathbb{N}}$ for $S_{p}$ is the restriction to $S_{p}$ of the $m^{\text {th }}$ coordinate functional on $\mathbb{K}^{\mathbb{N}}$, that is, $e_{m}^{\prime}=\left.f_{m}\right|_{S_{p}}$; Lemma 3.3 i) implies that this functional has norm 1 .

REMARK 3.7. The Banach space denoted by $S_{1}$ in 3.6 is the one which is usually called the Schreier space in the literature. Beauzamy and Lapresté formally introduced it in [6], building on ideas from Baernstein's thesis [4]; an introduction to this work, its context, and many further developments is given in [15, Chapter 0].

We are not aware of any previous studies of the Banach spaces $Z_{p}$ and $S_{p}$ for $p>1$.

ExAMPLE 3.8. Let $p \geqslant 1$. As we shall see in Corollary 5.6, $Z_{p}$ contains a subspace isomorphic to $\ell_{\infty}$. Hence $Z_{p}$ is non-separable and cannot, therefore, have a basis, so in particular $Z_{p}$ is not isomorphic to $S_{p}$. The purpose of this example is much simpler, namely to prove that $S_{p}$ is a proper subspace of $Z_{p}$. More precisely, we shall show that $z:=\left(n^{-\frac{1}{p}}\right)_{n \in \mathbb{N}} \in Z_{p} \backslash S_{p}$.

Suppose that $A=\left\{n_{1}<n_{2}<\cdots<n_{k}\right\} \subseteq \mathbb{N}$ is admissible. Since $z$ is decreasing and positive, we have

$$
\mu_{p}(z, A)^{p}=\sum_{j=1}^{k} \frac{1}{n_{j}} \leqslant \sum_{j=n_{1}}^{n_{1}+k-1} \frac{1}{j} \leqslant \sum_{j=n_{1}}^{2 n_{1}-1} \frac{1}{j} \leqslant n_{1} \cdot \frac{1}{n_{1}}=1
$$

so $z \in Z_{p}$ with $\|z\|_{Z_{p}} \leqslant 1$; in fact, $z$ is a unit vector because $\left\langle z, f_{1}\right\rangle=1$. On the other hand, $z \notin S_{p}$ because, for any $y=\sum_{n=1}^{m} \alpha_{n} e_{n} \in c_{00}$, the set $B:=\mathbb{N} \cap(m, 2 m+1]$ is admissible, and therefore

$$
\|z-y\|_{Z_{p}}^{p} \geqslant \mu_{p}(z-y, B)^{p}=\sum_{n=m+1}^{2 m+1} \frac{1}{n} \geqslant \int_{m+1}^{2 m+2} \frac{\mathrm{d} t}{t}=\log \frac{2 m+2}{m+1}=\log 2>0 .
$$

We shall next prove that the standard unit vector basis for $S_{p}$ is shrinking for each $p \geqslant 1$. As a consequence, we obtain a description of the bidual of $S_{p}$ in terms of $Z_{p}$. Our approach relies on the following well-known characterization of shrinking bases (e.g., see [1, Proposition 3.2.7]).

Lemma 3.9. A basis $\left(b_{n}\right)_{n \in \mathbb{N}}$ for a Banach space $X$ is shrinking if and only if every normalized block basic sequence of $\left(b_{n}\right)_{n \in \mathbb{N}}$ is a weakly null sequence in $X$.

The fact that the standard unit vector basis for $S_{1}$ is shrinking is well-known, although we have been unable to trace the original source of this result. We shall outline an elegant proof communicated to us by András Zsák.

Proposition 3.10. The standard unit vector basis $\left(e_{n}\right)_{n \in \mathbb{N}}$ for $S_{1}$ is shrinking. 
Proof. Each subset $A$ of $\mathbb{N}$ induces a functional $\omega_{A}$ on $c_{00}$ via the definition

$$
\left\langle x, \omega_{A}\right\rangle:=\sum_{n \in A}\left\langle x, f_{n}\right\rangle \quad\left(x \in c_{00}\right)
$$

and this functional is contractive with respect to the first Schreier norm if and only if the set $A$ is either empty or admissible. Thus, we may regard

$$
\Omega:=\left\{\omega_{A}: A=\emptyset \text { or } A \subseteq \mathbb{N} \text { admissible }\right\}
$$

as a subset of the closed unit ball of $S_{1}^{\prime}$, and we have

$$
\Omega=\left\{f \in S_{1}^{\prime}:\|f\|_{S_{1}^{\prime}} \leqslant 1 \text { and }\left\langle e_{n}, f\right\rangle \in\{0,1\}(n \in \mathbb{N})\right\}
$$

which implies that $\Omega$ is weak*-closed and hence weak*-compact by the Banach-Alaoglu Theorem.

For each $x \in S_{1}$, the mapping $U x: f \mapsto\langle x, f\rangle, \Omega \rightarrow \mathbb{K}$, is continuous with respect to the weak*-topology on $\Omega$, so it induces a mapping $U: S_{1} \rightarrow C(\Omega)$. Straightforward verifications show that $U$ is linear and contractive. Moreover, $U$ is bounded below by

$$
\varepsilon:= \begin{cases}\frac{1}{2} & \text { for } \mathbb{K}=\mathbb{R} \\ \frac{1}{4} & \text { for } \mathbb{K}=\mathbb{C}\end{cases}
$$

as we shall now prove. It suffices to show that, for each $x \in c_{00}$, there is a subset $A$ of $\mathbb{N}$, either admissible or empty, such that $\left|\left\langle x, \omega_{A}\right\rangle\right| \geqslant \varepsilon\|x\|_{S_{1}}$.

In the case where $x$ has real coordinates, take an admissible subset $B$ of $\mathbb{N}$ such that $\|x\|_{S_{1}}=\mu_{1}(x, B)$. The sets $B_{+}:=\left\{n \in B:\left\langle x, f_{n}\right\rangle>0\right\}$ and $B_{-}:=\left\{n \in B:\left\langle x, f_{n}\right\rangle<0\right\}$ are then admissible or empty, and they satisfy

$$
\|x\|_{S_{1}}=\mu_{1}(x, B)=\sum_{n \in B_{+}}\left\langle x, f_{n}\right\rangle-\sum_{n \in B_{-}}\left\langle x, f_{n}\right\rangle=\left|\left\langle x, \omega_{B_{+}}\right\rangle\right|+\left|\left\langle x, \omega_{B_{-}}\right\rangle\right|,
$$

so we conclude that $\left|\left\langle x, \omega_{A}\right\rangle\right| \geqslant\|x\|_{S_{1}} / 2$ holds for either $A:=B_{+}$or $A:=B_{-}$.

Now suppose that $x$ has complex coordinates and define $y:=\sum_{n=1}^{\infty} \operatorname{Re}\left\langle x, f_{n}\right\rangle e_{n}$ and $z:=\sum_{n=1}^{\infty} \operatorname{Im}\left\langle x, f_{n}\right\rangle e_{n}$. Then $x=y+\mathrm{i} z$, so that $\|x\|_{S_{1}} \leqslant\|y\|_{S_{1}}+\|z\|_{S_{1}}$ and thus either $\|y\|_{S_{1}} \geqslant\|x\|_{S_{1}} / 2$ or $\|z\|_{S_{1}} \geqslant\|x\|_{S_{1}} / 2$. We consider the first case only; the second is similar. As $y$ has real coordinates, the first part of the argument applies, yielding an admissible (or empty) set $A$ such that $\left|\left\langle y, \omega_{A}\right\rangle\right| \geqslant\|y\|_{S_{1}} / 2$, and consequently we have

$$
\left|\left\langle x, \omega_{A}\right\rangle\right|=\left|\left\langle y, \omega_{A}\right\rangle+\mathrm{i}\left\langle z, \omega_{A}\right\rangle\right| \geqslant\left|\left\langle y, \omega_{A}\right\rangle\right| \geqslant \frac{\|y\|_{S_{1}}}{2} \geqslant \frac{\|x\|_{S_{1}}}{4},
$$

as required.

In conclusion, $U$ is an isomorphism of $S_{1}$ onto its image inside $C(\Omega)$. Now let $\left(u_{n}\right)_{n \in \mathbb{N}}$ be a normalized block basic sequence of $\left(e_{n}\right)_{n \in \mathbb{N}}$ in $S_{1}$. By Lemma 3.9, we must prove that $\left(u_{n}\right)_{n \in \mathbb{N}}$ is weakly null in $S_{1}$. This is equivalent to $\left(U u_{n}\right)_{n \in \mathbb{N}}$ being weakly null in $C(\Omega)$ because the weak topology on the image of $U$ in $C(\Omega)$ is just the restriction of the weak topology on $C(\Omega)$. For each $\omega_{A} \in \Omega$, we have $\left(U u_{n}\right)\left(\omega_{A}\right)=\left\langle u_{n}, \omega_{A}\right\rangle=0$ eventually because $A \subseteq \mathbb{N}$ is finite, while $\left(u_{n}\right)_{n \in \mathbb{N}}$ is a block basic sequence. Hence $\left(U u_{n}\right)_{n \in \mathbb{N}}$ converges pointwise to 0 on $\Omega$. As this sequence is also norm-bounded, [16, Corollary IV.6.4] implies that it is weakly null in $C(\Omega)$, as required. 
The proof above does not work for $p>1$, but fortunately an easier route is available for such $p$. This relies on the following result.

Lemma 3.11. Let $p \geqslant 1$, and let $\left(u_{n}\right)_{n \in \mathbb{N}}$ be a normalized block basic sequence of the standard unit vector basis $\left(e_{n}\right)_{n \in \mathbb{N}}$ for $S_{p}$. Then the operator

$$
T: \sum_{n=1}^{\infty} \alpha_{n} e_{n} \mapsto \sum_{n=1}^{\infty} \alpha_{n} u_{n}, \quad\left(c_{00},\|\cdot\|_{\ell_{p}}\right) \rightarrow S_{p},
$$

is contractive, and therefore it extends uniquely to a contractive operator $T: \ell_{p} \rightarrow S_{p}$.

Proof. Choose integers $0=M_{0}<M_{1}<\cdots<M_{n-1}<M_{n}<\cdots$ such that

$$
u_{n} \in \operatorname{span}\left\{e_{j}: M_{n-1}<j \leqslant M_{n}\right\} \quad(n \in \mathbb{N}) .
$$

Given an admissible subset $A$ of $\mathbb{N}$, define $A_{n}:=A \cap\left(M_{n-1}, M_{n}\right]$ for each $n \in \mathbb{N}$. Then $A$ is the disjoint union of $\left(A_{n}\right)_{n \in \mathbb{N}}$, and each of the sets $A_{n}$ is either empty or admissible. Hence, for $x=\sum_{n=1}^{\infty} \alpha_{n} e_{n} \in c_{00}$, we have

$$
\begin{aligned}
\|x\|_{\ell_{p}}^{p} & =\sum_{n=1}^{\infty}\left|\alpha_{n}\right|^{p} \geqslant \sum_{n=1}^{\infty}\left|\alpha_{n}\right|^{p} \mu_{p}\left(u_{n}, A_{n}\right)^{p}=\sum_{n=1}^{\infty} \mu_{p}\left(\alpha_{n} u_{n}, A_{n}\right)^{p} \\
& =\sum_{n=1}^{\infty} \mu_{p}\left(\sum_{j=1}^{\infty} \alpha_{j} u_{j}, A_{n}\right)^{p}=\sum_{n=1}^{\infty} \mu_{p}\left(T x, A_{n}\right)^{p}=\mu_{p}(T x, A)^{p},
\end{aligned}
$$

and the result follows by taking the supremum over all admissible sets $A$.

COROLlaRY 3.12. For each $p>1$, the standard unit vector basis $\left(e_{n}\right)_{n \in \mathbb{N}}$ for $S_{p}$ is shrinking.

Proof. Let $\left(u_{n}\right)_{n \in \mathbb{N}}$ be a normalized block basic sequence of $\left(e_{n}\right)_{n \in \mathbb{N}}$ in $S_{p}$, and consider the contractive operator $T: \ell_{p} \rightarrow S_{p}$ from Lemma 3.11. Since $p>1$, the standard unit vector basis $\left(e_{n}\right)_{n \in \mathbb{N}}$ is a weakly null sequence in $\ell_{p}$, so the boundedness of $T$ implies that the sequence $\left(T e_{n}\right)_{n \in \mathbb{N}}=\left(u_{n}\right)_{n \in \mathbb{N}}$ is weakly null in $S_{p}$. Hence the result follows from Lemma 3.9 .

Lemma 3.13. Let $p \geqslant 1$. Then $\|x\|_{\operatorname{bip}\left(S_{p}\right)}=\|x\|_{Z_{p}}$ for each $x \in \mathbb{K}^{\mathbb{N}}$, and so $\operatorname{bip}\left(S_{p}\right)=Z_{p}$.

Proof. Comparing the definitions 2.1 and (1.4), we see that

$$
\|x\|_{\operatorname{bip}\left(S_{p}\right)}=\sup \left\{\left\|P_{m} x\right\|_{S_{p}}: m \in \mathbb{N}\right\} \quad\left(x \in \mathbb{K}^{\mathbb{N}}\right) .
$$

Hence the inequality $\|x\|_{\operatorname{bip}\left(S_{p}\right)} \leqslant\|x\|_{Z_{p}}$ follows from Lemma 3.3 iii) whenever $x \in Z_{p}$; otherwise (that is, for $x \in \mathbb{K}^{\mathbb{N}} \backslash Z_{p}$ ) it is trivial.

Conversely, let $A$ be an admissible subset of $\mathbb{N}$. Taking $m:=\max A \in \mathbb{N}$, we obtain

$$
\mu_{p}(x, A)=\mu_{p}\left(P_{m} x, A\right) \leqslant\left\|P_{m} x\right\|_{S_{p}} \leqslant\|x\|_{\text {bip }\left(S_{p}\right)},
$$

and therefore $\|x\|_{Z_{p}} \leqslant\|x\|_{\operatorname{bip}\left(S_{p}\right)}$.

3.14. The unrestricted Schreier space and the bidual of $S_{p}$. For each $p \geqslant 1$, we can combine the results of Proposition 3.10, Corollary 3.12, Lemma 3.13, and $\$ 2.4$ to obtain 
a commutative diagram

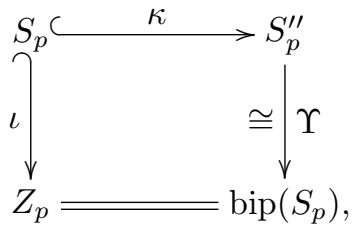

where $\iota$ is the natural inclusion, $\kappa$ is the canonical embedding, and $\Upsilon$ is the isometric isomorphism from $\$ 2.4$. Thus, if we identify the bidual of $S_{p}$ with $\operatorname{bip}\left(S_{p}\right)$ via $\Upsilon$, then $\kappa=\iota$, that is, the canonical embedding of $S_{p}$ into its bidual becomes the natural inclusion of $S_{p}$ into $Z_{p}$.

3.15. Shift operators. Let $\sigma: \mathbb{N} \rightarrow \mathbb{N}$ be strictly increasing. We associate with $\sigma$ two operators on $c_{00}$, the left shift $\Lambda_{\sigma}$ and the right shift $R_{\sigma}$, given by

$$
\Lambda_{\sigma} x:=\sum_{n=1}^{\infty} \alpha_{\sigma(n)} e_{n} \quad \text { and } \quad R_{\sigma} x:=\sum_{n=1}^{\infty} \alpha_{n} e_{\sigma(n)} \quad\left(x=\sum_{n=1}^{\infty} \alpha_{n} e_{n} \in c_{00}\right) .
$$

They are clearly linear, and $\Lambda_{\sigma} R_{\sigma}=I_{c_{00}}$, while $R_{\sigma} \Lambda_{\sigma}$ is the restriction to $c_{00}$ of the natural projection $P_{\sigma(\mathbb{N})}$ associated with the set $\sigma(\mathbb{N})$.

Our interest in these operators lies primarily in understanding when they extend to bounded operators on $S_{p}$. In order to treat both cases simultaneously (and for later use, see the proof of Proposition 4.20 below), we introduce the mixed shift operator $\Xi_{\tau, \omega}$ associated with a pair of injective mappings $\tau, \omega: \mathbb{N} \rightarrow \mathbb{N}$ by

$$
\Xi_{\tau, \omega}: \quad \sum_{n=1}^{\infty} \alpha_{n} e_{n} \mapsto \sum_{n=1}^{\infty} \alpha_{\tau(n)} e_{\omega(n)}, \quad c_{00} \rightarrow c_{00} .
$$

Again, $\Xi_{\tau, \omega}$ is clearly linear, and writing $\iota$ for the identity mapping $n \mapsto n, \mathbb{N} \rightarrow \mathbb{N}$, we see that $\Lambda_{\sigma}=\Xi_{\sigma, \iota}$ and $R_{\sigma}=\Xi_{\iota, \sigma}$.

LEMMA 3.16. Let $p \geqslant 1$, let $\tau, \omega: \mathbb{N} \rightarrow \mathbb{N}$ be injective, and suppose that there is a constant $K \in \mathbb{N}$ such that $\omega(n) \leqslant K \tau(n)$ for each $n \in \mathbb{N}$. Then $\left\|\Xi_{\tau, \omega} x\right\|_{S_{p}}^{p} \leqslant K\|x\|_{S_{p}}^{p}$ for each $x \in c_{00}$, and thus $\Xi_{\tau, \omega}$ extends uniquely to a bounded operator on $S_{p}$ of norm at most $K^{\frac{1}{p}}$. Proof. Let $x \in c_{00}$ and an admissible subset $A$ of $\mathbb{N}$ be given. If $A \cap \omega(\mathbb{N})=\emptyset$, then $\mu_{p}\left(\Xi_{\tau, \omega} x, A\right)=0$. Otherwise choose $B \subseteq \mathbb{N}$ such that $A \cap \omega(\mathbb{N})=\omega(B)$, and let $m:=\min \tau(B)$. Being a non-empty subset of the admissible set $A$, the set $\omega(B)$ is itself admissible, and this, together with our assumptions on $\omega$ and $\tau$, implies that

$$
\operatorname{card} B=\operatorname{card} \omega(B) \leqslant \min \omega(B) \leqslant K \min \tau(B)=K m,
$$

so we can express $B$ as the union of $K$ disjoint sets $B_{1}, \ldots, B_{K}$, say, each having at most $m$ elements. Thus each of the sets $\tau\left(B_{1}\right), \ldots, \tau\left(B_{K}\right)$ is admissible (or empty), and therefore we have

$$
\begin{aligned}
K\|x\|_{S_{p}}^{p} & \geqslant \sum_{j=1}^{K} \mu_{p}\left(x, \tau\left(B_{j}\right)\right)^{p}=\mu_{p}(x, \tau(B))^{p}=\sum_{n \in B}\left|\left\langle x, f_{\tau(n)}\right\rangle\right|^{p} \\
& =\sum_{n \in B}\left|\left\langle\Xi_{\tau, \omega} x, f_{\omega(n)}\right\rangle\right|^{p}=\mu_{p}\left(\Xi_{\tau, \omega} x, \omega(B)\right)^{p}=\mu_{p}\left(\Xi_{\tau, \omega} x, A\right)^{p} .
\end{aligned}
$$

Now the result follows by taking the supremum over all admissible sets $A$. 
COROllary 3.17. Let $p \geqslant 1$, and let $\sigma: \mathbb{N} \rightarrow \mathbb{N}$ be strictly increasing. Then:

(i) $\left\|\Lambda_{\sigma} x\right\|_{S_{p}} \leqslant\|x\|_{S_{p}}$ for each $x \in c_{00}$, so that $\Lambda_{\sigma}$ extends uniquely to a contractive operator on $S_{p}$, no matter what $\sigma$ is;

(ii) $R_{\sigma}$ is a bounded operator on $\left(c_{00},\|\cdot\|_{S_{p}}\right)$ if and only if there is a constant $K \in \mathbb{N}$ such that $\sigma(n) \leqslant K n$ for each $n \in \mathbb{N}$. In the positive case, $R_{\sigma}$ extends uniquely to a bounded operator of norm at most $K^{\frac{1}{p}}$ on $S_{p}$.

Proof. To prove (i), recall from $\$ 3.15$ that $\Lambda_{\sigma}=\Xi_{\sigma, \iota}$. Since $\sigma$ is strictly increasing, we have $\sigma(n) \geqslant n=\iota(n)$ for each $n \in \mathbb{N}$, so that the condition in Lemma 3.16 is satisfied with $K=1$, and therefore $\Lambda_{\sigma}$ extends to a bounded operator on $S_{p}$ with norm at most 1 .

The implication ' $\Leftarrow$ ' in (ii) and the final clause follow in a similar fashion, so it only remains to prove ' $\Rightarrow$ ' in (ii). Thus, suppose that $R_{\sigma}:\left(c_{00},\|\cdot\|_{S_{p}}\right) \rightarrow\left(c_{00},\|\cdot\|_{S_{p}}\right)$ is bounded, let $n \in \mathbb{N}$ be given, and consider the unit vector $z:=\left(m^{-\frac{1}{p}}\right)_{m \in \mathbb{N}} \in Z_{p}$ from Example 3.8. If we define $N:=n+\sigma(n)-1$ and $A:=\mathbb{N} \cap[n, N]$, then the set $\sigma(A)$ is admissible, and Lemma 3.3 iii) implies that $P_{A} z \in c_{00}$ with norm at most 1 , so that

$$
\begin{aligned}
\left\|R_{\sigma}\right\|^{p} & \geqslant\left\|R_{\sigma} P_{A} z\right\|_{S_{p}}^{p} \geqslant \mu_{p}\left(R_{\sigma} P_{A} z, \sigma(A)\right)^{p}=\sum_{m=n}^{N}\left|\left\langle R_{\sigma} P_{A} z, f_{\sigma(m)}\right\rangle\right|^{p} \\
& =\sum_{m=n}^{N}\left|\left\langle z, f_{m}\right\rangle\right|^{p}=\sum_{m=n}^{N} \frac{1}{m} \geqslant \int_{n}^{N+1} \frac{\mathrm{d} t}{t}=\log \frac{N+1}{n}=\log \left(1+\frac{\sigma(n)}{n}\right)
\end{aligned}
$$

from which we deduce that $\left(e^{\left\|R_{\sigma}\right\|^{p}}-1\right) n \geqslant \sigma(n)$; hence any integer $K \geqslant e^{\left\|R_{\sigma}\right\|^{p}}-1$ has the required property.

The following well-known observation will be called upon repeatedly in the sequel.

REMARK 3.18. Let $X$ and $Y$ be normed spaces, and let $\Gamma_{1}: X \rightarrow Y$ and $\Gamma_{2}: Y \rightarrow X$ be bounded operators with $\Gamma_{1} \Gamma_{2}=I_{Y}$. Then $P:=\Gamma_{2} \Gamma_{1}$ is a bounded idempotent operator on $X$ whose image is isomorphic to $Y$. More precisely, we have $\operatorname{im} P=\operatorname{im} \Gamma_{2}$, and the 'corestriction' of $\Gamma_{2}$ to im $P$ (that is, the operator $y \mapsto \Gamma_{2} y, Y \rightarrow \operatorname{im} P$ ) is an isomorphism; its inverse is the restriction of $\Gamma_{1}$ to $\mathrm{im} P$.

COROLlary 3.19. For each $p \geqslant 1$, the $p^{\text {th }}$ Schreier space $S_{p}$ is isomorphic to its Cartesian square $S_{p} \oplus S_{p}$.

Proof. Consider the strictly increasing mappings $\alpha, \beta: \mathbb{N} \rightarrow \mathbb{N}$ given by

$$
\alpha(n):=2 n-1 \quad \text { and } \quad \beta(n):=2 n \quad(n \in \mathbb{N}) .
$$

Corollary 3.17 shows that the associated shifts $\Lambda_{\alpha}, R_{\alpha}, \Lambda_{\beta}$, and $R_{\beta}$ extend to bounded operators on $S_{p}$. Consequently, the identities stated in 3.15 immediately after 3.2 together with Remark 3.18 imply that $Q_{\alpha}:=R_{\alpha} \Lambda_{\alpha}$ and $Q_{\beta}:=R_{\beta} \Lambda_{\beta}$ are bounded idempotent operators on $S_{p}$ whose images are isomorphic to $S_{p}$, and therefore we have

$$
S_{p} \oplus S_{p} \cong \operatorname{im} Q_{\alpha} \oplus \operatorname{im} Q_{\beta} \cong S_{p}
$$

where the final isomorphism follows from the fact that $\operatorname{im} Q_{\beta}=\operatorname{ker} Q_{\alpha}$, so that $\operatorname{im} Q_{\alpha}$ and $\operatorname{im} Q_{\beta}$ are closed, complementary subspaces of $S_{p}$. 
3.20. Further results. We conclude this section by listing a few results from the literature on Schreier spaces.

(i) Alspach and Argyros [2] have generalized the notion of admissible subset of $\mathbb{N}$ by introducing the Schreier families of finite subsets of $\mathbb{N}$. The Schreier families are indexed by the countable ordinals; the first one is exactly the collection of all admissible subsets of $\mathbb{N}$. In analogy with the definition of the $p^{\text {th }}$ Schreier space based on the admissible subsets of $\mathbb{N}$ (as presented in this section), one can associate a $p^{\text {th }}$ Schreier space with each Schreier family. There is a substantial body of theory concerning these spaces in the case $p=1$; the case $p>1$ appears to be unchartered territory.

(ii) Gasparis and Leung [19] have studied the complemented subspaces of the first Schreier space $S_{1}$ (as well as those of the generalized Schreier spaces of Alspach and Argyros described above). Their main result is that there are uncountably many non-isomorphic subspaces among those of the form $\overline{\operatorname{span}}\left\{e_{n}: n \in A\right\}$, where $A$ is an infinite subset of $\mathbb{N}$.

4. Introduction to the James-Schreier spaces. We shall now amalgamate the definitions of the $p^{\text {th }}$ James space and the $p^{\text {th }}$ Schreier space for each $p \geqslant 1$. Recall from $\$ 2.1$ that, by definition, the $p^{\text {th }}$ James norm of $x \in \mathbb{K}^{\mathbb{N}}$ is the supremum of $\nu_{p}(x, A)$ over all finite subsets $A$ of $\mathbb{N}$; our idea is to consider only those sets $A$ which satisfy an admissibility-like condition.

Definition 4.1. A subset $A$ of $\mathbb{N}$ is permissible if

$$
2 \leqslant \operatorname{card} A \leqslant 1+\min A .
$$

Thus a permissible set must contain at least two elements and be finite, and for $A=\left\{n_{1}<n_{2}<\cdots<n_{k+1}\right\} \subseteq \mathbb{N}$, where $k \in \mathbb{N}$, we have

$$
A \text { is permissible } \Leftrightarrow k \leqslant n_{1} \text {. }
$$

Definition 4.2. Let $1 \leqslant p<\infty$. For $x=\left(\alpha_{n}\right)_{n \in \mathbb{N}} \in \mathbb{K}^{\mathbb{N}}$, we define

$$
\begin{aligned}
\|x\|_{W_{p}}: & =\sup \left\{\nu_{p}(x, A): A \subseteq \mathbb{N} \text { is permissible }\right\} \\
& =\sup \left\{\left(\sum_{j=1}^{k}\left|\alpha_{n_{j}}-\alpha_{n_{j+1}}\right|^{p}\right)^{\frac{1}{p}}: k, n_{1}, \ldots, n_{k+1} \in \mathbb{N}, k \leqslant n_{1}<\cdots<n_{k+1}\right\},
\end{aligned}
$$

and we then let $W_{p}:=\left\{x \in c_{0}:\|x\|_{W_{p}}<\infty\right\}$. We call $\|\cdot\|_{W_{p}}$ the $p^{\text {th }}$ James-Schreier norm and $W_{p}$ the $p^{\text {th }}$ unrestricted James-Schreier space.

The following lemma is the James-Schreier counterpart of Lemma 3.3, we omit its routine proof.

Lemma 4.3. Let $p \geqslant 1$ and $m \in \mathbb{N}$. Then:

(i) the restriction to $W_{p}$ of the $m^{\text {th }}$ coordinate functional $f_{m}$ given by 1.2 is bounded with norm 1;

(ii) $W_{p}$ is an invariant subspace for the $m^{\text {th }}$ natural projection $P_{m}$ given by (1.4), and the restriction of $P_{m}$ to $W_{p}$ is bounded with norm 1; 
(iii) $W_{p}$ is a Banach space with respect to the coordinatewise defined operations inherited from $\mathbb{K}^{\mathbb{N}}$ and the norm $\|\cdot\|_{W_{p}}$.

We shall next show that, for each $p \geqslant 1, W_{p}$ contains a complemented subspace which is isomorphic to the unrestricted Schreier space $Z_{p}$. In the proof we require the following well-known inequality

$$
|\alpha+\beta|^{p} \leqslant 2^{p-1}\left(|\alpha|^{p}+|\beta|^{p}\right) \quad(p \geqslant 1, \alpha, \beta \in \mathbb{K})
$$

which is an easy consequence of the convexity of the function $t \mapsto t^{p},[0, \infty) \rightarrow[0, \infty)$.

Definition 4.4. For $x=\left(\alpha_{n}\right)_{n \in \mathbb{N}} \in \mathbb{K}^{\mathbb{N}}$, let

$$
\Phi x:=\left(0, \alpha_{1}, 0, \alpha_{2}, 0, \alpha_{3}, \ldots\right) \in \mathbb{K}^{\mathbb{N}} \quad \text { and } \quad \Psi x:=\left(\alpha_{2 n}-\alpha_{2 n-1}\right)_{n \in \mathbb{N}} \in \mathbb{K}^{\mathbb{N}} .
$$

Proposition 4.5. Let $p \geqslant 1$. The first equation in 4.2 defines a bounded operator $\Phi$ from $Z_{p}$ to $W_{p}$ of norm at most $2^{1+\frac{1}{p}}$, while the second defines a bounded operator $\Psi$ from $W_{p}$ to $Z_{p}$ of norm 1 . These operators satisfy $\Psi \Phi=I_{Z_{p}}$, and hence $\Phi \Psi$ is idempotent with

$$
\operatorname{im}(\Phi \Psi)=\left\{\left(\alpha_{n}\right)_{n \in \mathbb{N}} \in W_{p}: \alpha_{2 n-1}=0 \quad(n \in \mathbb{N})\right\} \cong Z_{p} .
$$

Proof. Considered as mappings on $\mathbb{K}^{\mathbb{N}}, \Phi$ and $\Psi$ are clearly both linear, so to establish the first part of the proposition, it suffices to show that

(i) $\Phi x \in W_{p}$ with $\|\Phi x\|_{W_{p}} \leqslant 2^{1+\frac{1}{p}}\|x\|_{Z_{p}}$ for each $x \in Z_{p}$; and

(ii) $\Psi x \in Z_{p}$ with $\|\Psi x\|_{Z_{p}} \leqslant\|x\|_{W_{p}}$ for each $x \in W_{p}$, with equality for some $x \in W_{p}$.

To prove (i), let $x \in Z_{p}$ be given. Lemma 3.3 (ii) implies that $x \in c_{0}$, and therefore $\Phi x \in c_{0}$ by the definition of $\Phi$. Now suppose that $A=\left\{n_{1}<n_{2}<\cdots<n_{k+1}\right\}$ is a permissible subset of $\mathbb{N}$. If $A \cap 2 \mathbb{N}=\emptyset$, then $\nu_{p}(\Phi x, A)=0$. Otherwise we choose $\ell, m_{1}, \ldots, m_{\ell} \in \mathbb{N}$ such that $A \cap 2 \mathbb{N}=\left\{2 m_{1}<2 m_{2}<\cdots<2 m_{\ell}\right\}$. Using the inequality 4.1, we obtain

$$
\begin{aligned}
\nu_{p}(\Phi x, A)^{p} & =\sum_{j=1}^{k}\left|\left\langle\Phi x, f_{n_{j}}-f_{n_{j+1}}\right\rangle\right|^{p} \leqslant 2^{p-1} \sum_{j=1}^{k}\left(\left|\left\langle\Phi x, f_{n_{j}}\right\rangle\right|^{p}+\left|\left\langle\Phi x, f_{n_{j+1}}\right\rangle\right|^{p}\right) \\
& \leqslant 2^{p} \mu_{p}(\Phi x, A)^{p}=2^{p} \mu_{p}(\Phi x, A \cap 2 \mathbb{N})^{p}=2^{p} \mu_{p}(x, B)^{p},
\end{aligned}
$$

where we have introduced $B:=\left\{m_{1}<m_{2}<\cdots<m_{\ell}\right\} \subseteq \mathbb{N}$. Now let $h:=\min \left\{m_{1}, \ell\right\}$, $B_{1}:=\left\{m_{1}<m_{2}<\cdots<m_{h}\right\} \subseteq \mathbb{N}$, and $B_{2}:=B \backslash B_{1} \subseteq \mathbb{N}$; we claim that

$$
\mu_{p}\left(x, B_{j}\right) \leqslant\|x\|_{Z_{p}} \quad(j=1,2) .
$$

This is immediate for $j=1$ because the definition of $h$ ensures that $B_{1}$ is admissible. If $h=\ell$, then $B_{2}=\emptyset$, so that 4.4 is satisfied for $j=2$ by convention. Otherwise $h=m_{1}<\ell$ and $B_{2}=\left\{m_{h+1}<m_{h+2}<\cdots<m_{\ell}\right\}$, so that $B_{2}$ is admissible because

$$
\begin{aligned}
\min B_{2} & =m_{h+1} \geqslant m_{h}+1 \geqslant m_{h-1}+2 \geqslant \cdots \geqslant m_{1}+h \\
& =2 m_{1} \geqslant \min A \geqslant(\operatorname{card} A)-1 \geqslant \ell-1 \geqslant \ell-h=\operatorname{card} B_{2},
\end{aligned}
$$

and (4.4) for $j=2$ follows. Since $B$ is the disjoint union of $B_{1}$ and $B_{2}$, we conclude that

$$
\mu_{p}(x, B)^{p}=\mu_{p}\left(x, B_{1}\right)^{p}+\mu_{p}\left(x, B_{2}\right)^{p} \leqslant 2\|x\|_{Z_{p}}^{p} .
$$


Substituting this into 4.3 and then taking the supremum over all permissible sets $A$, we see that (i) is satisfied.

To prove (ii), let $x \in W_{p}$, and suppose that $A=\left\{n_{1}<n_{2}<\cdots<n_{k}\right\} \subseteq \mathbb{N}$ is admissible, so that $k \leqslant n_{1}$. Then the set $B:=\left\{2 n_{j}-1,2 n_{j}: 1 \leqslant j \leqslant k\right\} \subseteq \mathbb{N}$ is permissible because card $B=2 k \leqslant 2 n_{1}=1+\min B$, and consequently we have

$$
\begin{aligned}
\|x\|_{W_{p}}^{p} & \geqslant \nu_{p}(x, B)^{p} \\
& =\sum_{j=1}^{k-1}\left(\left|\left\langle x, f_{2 n_{j}-1}-f_{2 n_{j}}\right\rangle\right|^{p}+\left|\left\langle x, f_{2 n_{j}}-f_{2 n_{j+1}-1}\right\rangle\right|^{p}\right)+\left|\left\langle x, f_{2 n_{k}-1}-f_{2 n_{k}}\right\rangle\right|^{p} \\
& \geqslant \sum_{j=1}^{k}\left|\left\langle x, f_{2 n_{j}-1}-f_{2 n_{j}}\right\rangle\right|^{p}=\sum_{j=1}^{k}\left|\left\langle\Psi x, f_{n_{j}}\right\rangle\right|^{p}=\mu_{p}(\Psi x, A)^{p} .
\end{aligned}
$$

Taking the supremum over all admissible sets $A$, we conclude that $\|x\|_{W_{p}} \geqslant\|\Psi x\|_{Z_{p}}$, as required. Since $\Psi e_{1}=-e_{1}$ and $e_{1}$ is a unit vector in both $W_{p}$ and $Z_{p}$, equality does occur. This completes the proof of (ii).

Direct application of the definitions 4.2 shows that $\Psi \Phi=I_{Z_{p}}$, so Remark 3.18 implies that $P:=\Phi \Psi$ is a bounded idempotent operator with image isomorphic to $Z_{p}$, and clearly

$$
\operatorname{im} P \subseteq\left\{\left(\alpha_{n}\right)_{n \in \mathbb{N}} \in W_{p}: \alpha_{2 n-1}=0 \quad(n \in \mathbb{N})\right\} .
$$

Conversely, suppose that $x=\left(\alpha_{n}\right)_{n \in \mathbb{N}} \in W_{p}$ satisfies $\alpha_{2 n-1}=0$ for each $n \in \mathbb{N}$; then we have

$$
\operatorname{im} P \ni P x=\Phi \Psi x=\Phi\left(\alpha_{2 n}\right)_{n \in \mathbb{N}}=\left(0, \alpha_{2}, 0, \alpha_{4}, 0, \alpha_{6}, \ldots\right)=x,
$$

as required.

REMARK 4.6. We can now give an easy proof of Lemma 3.3 iv. Indeed, Proposition 4.5 implies that $Z_{p}$ is isomorphic to a complemented subspace of $W_{p}$, which is a Banach space by Lemma 4.3 iii). Complemented subspaces are automatically closed, hence complete, and therefore $Z_{p}$ is also complete.

Proposition 4.7. For each $p \geqslant 1$, the sequence $\left(e_{n}\right)_{n \in \mathbb{N}}$ given by $(1.1$ is a monotone basic sequence in $W_{p}$.

Proof. Lemma 4.3 ii implies that $\left\|P_{m} x\right\|_{W_{p}} \leqslant\|x\|_{W_{p}}$ for each $x \in c_{00}$ and $m \in \mathbb{N}$, and hence the result follows from the monotone version of Banach's fundamental characterization of basic sequences (e.g., see [27, Proposition 1.a.3] or [30, Corollary 4.1.25]).

4.8. The restricted James-Schreier spaces. Lemma 4.3 iii and Proposition 4.7 imply that

$$
V_{p}:=\overline{\operatorname{span}}\left\{e_{n}: n \in \mathbb{N}\right\} \subseteq W_{p}
$$

is a Banach space with a monotone basis $\left(e_{n}\right)_{n \in \mathbb{N}}$ for each $p \geqslant 1$. We call $V_{p}$ the $p^{\text {th }}$ (restricted) James-Schreier space and refer to $\left(e_{n}\right)_{n \in \mathbb{N}}$ as its standard basis. As in the case of the Schreier spaces, we omit the adjective 'restricted', unless confusion with $W_{p}$ seems likely. We write $\|\cdot\|_{V_{p}}$ for the restriction to $V_{p}$ of the norm $\|\cdot\|_{W_{p}}$. 
Note that the $m^{\text {th }}$ biorthogonal functional associated with the standard basis $\left(e_{n}\right)_{n \in \mathbb{N}}$ for $V_{p}$ is the restriction to $V_{p}$ of the $m^{\text {th }}$ coordinate functional on $\mathbb{K}^{\mathbb{N}}$, that is, $e_{m}^{\prime}=\left.f_{m}\right|_{V_{p}}$; this functional has norm 1 by Lemma 4.3 i).

REMARK 4.9. (i) Only the first two basis vectors $e_{1}$ and $e_{2}$ are normalized in the $p^{\text {th }}$ James-Schreier norm; for $n \geqslant 3$, we have $\left\|e_{n}\right\|_{V_{p}}=\nu_{p}\left(e_{n},\{n-1, n, n+1\}\right)=2^{\frac{1}{p}}$.

(ii) The standard basis $\left(e_{n}\right)_{n \in \mathbb{N}}$ for $V_{p}$ is not unconditional because $\chi_{[1,2 n]}$ is a unit vector for each $n \in \mathbb{N}$, whereas

$$
\left\|\sum_{j=1}^{2 n}(-1)^{j} e_{j}\right\|_{V_{p}}=\nu_{p}\left(\sum_{j=1}^{2 n}(-1)^{j} e_{j},\{n, n+1, \ldots, 2 n\}\right)=2 n^{\frac{1}{p}} \rightarrow \infty \quad \text { as } \quad n \rightarrow \infty .
$$

We shall prove a much stronger result in Section 6 .

We observe next that Proposition 4.5 carries over to the restricted spaces.

Proposition 4.10. Let $p \geqslant 1$. The first equation in 4.2 defines a bounded operator $\Phi$ from $S_{p}$ to $V_{p}$ of norm at most $2^{1+\frac{1}{p}}$, while the second defines a bounded operator $\Psi$ from $V_{p}$ to $S_{p}$ of norm 1 . These operators satisfy $\Psi \Phi=I_{S_{p}}$, and hence $\Phi \Psi$ is idempotent with

$$
\operatorname{im}(\Phi \Psi)=\overline{\operatorname{span}}\left\{e_{2 n}: n \in \mathbb{N}\right\} \cong S_{p} .
$$

Proof. The operators $\Phi$ and $\Psi$ given by 4.2 clearly leave $c_{00}$ invariant. Since $c_{00}$ is dense in both $S_{p}$ and $V_{p}$, and $\Phi$ and $\Psi$ are bounded on the unrestricted spaces $Z_{p}$ and $W_{p}$, we conclude that $\Phi\left(S_{p}\right) \subseteq V_{p}$ and $\Psi\left(V_{p}\right) \subseteq S_{p}$. The result is now easy to deduce from Proposition 4.5 .

The following example is the James-Schreier analogue of Example 3.8 .

EXAMPLE 4.11. Let $p \geqslant 1$. Using the vector $z:=\left(n^{-\frac{1}{p}}\right)_{n \in \mathbb{N}} \in Z_{p} \backslash S_{p}$ from Example 3.8 . we can easily verify that $V_{p}$ is a proper subspace of $W_{p}$. Indeed, Proposition 4.5 implies that $y:=\Phi z \in W_{p}$, but $y \notin V_{p}$ because if it were, $\Psi y \in S_{p}$ by Proposition 4.10 . contradicting that $\Psi y=z \notin S_{p}$.

The much stronger conclusion that $W_{p} ¥ V_{p}$ will follow from Section 5 .

We shall next show that the standard basis for $V_{p}$ is shrinking whenever $p>1$; this will enable us to describe the bidual of $V_{p}$ in terms of $W_{p}$.

Proposition 4.12. For each $p>1$, the standard basis $\left(e_{n}\right)_{n \in \mathbb{N}}$ for $V_{p}$ is shrinking.

The proof of this result is identical to that of Corollary 3.12 provided that the operator $T$ from Lemma 3.11 is replaced with the operator $U$ from the following lemma throughout.

LEMma 4.13. Let $p \geqslant 1$, and let $\left(u_{n}\right)_{n \in \mathbb{N}}$ be a normalized block basic sequence of the standard basis $\left(e_{n}\right)_{n \in \mathbb{N}}$ for $V_{p}$. Then the operator

$$
U: \sum_{n=1}^{\infty} \alpha_{n} e_{n} \mapsto \sum_{n=1}^{\infty} \alpha_{n} u_{n}, \quad\left(c_{00},\|\cdot\|_{\ell_{p}}\right) \rightarrow V_{p},
$$

is bounded, and therefore it extends uniquely to a bounded operator $U: \ell_{p} \rightarrow V_{p}$.

Proof. As in the proof of Lemma 3.11, choose integers $0=M_{0}<M_{1}<\cdots<M_{n}<\cdots$ such that $u_{n} \in \operatorname{span}\left\{e_{j}: M_{n-1}<j \leqslant M_{n}\right\}$ for each $n \in \mathbb{N}$. Given a permissible subset $A$ 
of $\mathbb{N}$, take natural numbers $k$ and $n_{1}<n_{2}<\cdots<n_{k}$ such that

$$
A \cap\left(M_{n-1}, M_{n}\right] \neq \emptyset \Leftrightarrow n \in\left\{n_{1}, n_{2}, \ldots, n_{k}\right\} \quad(n \in \mathbb{N}),
$$

and define $A_{j}:=A \cap\left(M_{n_{j}-1}, M_{n_{j}}\right]$ for each $j \in\{1, \ldots, k\}$. Then $A$ is the disjoint union of the sets $A_{1}, \ldots, A_{k}$, each of which is either a singleton or permissible, and for each $x=\sum_{n=1}^{\infty} \alpha_{n} e_{n} \in c_{00}$, we have

$$
\begin{aligned}
\nu_{p}(U x, A)^{p} & =\sum_{j=1}^{k} \nu_{p}\left(U x, A_{j}\right)^{p}+\sum_{j=1}^{k-1}\left|\left\langle U x, f_{\max A_{j}}-f_{\min A_{j+1}}\right\rangle\right|^{p} \\
\text { by } & \leqslant \sum_{j=1}^{\leqslant} \nu_{p}\left(\alpha_{n_{j}} u_{n_{j}}, A_{j}\right)^{p}+2^{p-1} \sum_{j=1}^{k-1}\left(\left|\left\langle U x, f_{\max A_{j}}\right\rangle\right|^{p}+\left|\left\langle U x, f_{\min A_{j+1}}\right\rangle\right|^{p}\right) \\
\leqslant & \sum_{j=1}^{k}\left|\alpha_{n_{j}}\right|^{p}\left\|u_{n_{j}}\right\|_{V_{p}}^{p} \\
& \quad+2^{p-1} \sum_{j=1}^{k-1}\left(\left|\alpha_{n_{j}}\right|^{p}\left|\left\langle u_{n_{j}}, f_{\max A_{j}}\right\rangle\right|^{p}+\left|\alpha_{n_{j+1}}\right|^{p}\left|\left\langle u_{n_{j+1}}, f_{\min A_{j+1}}\right\rangle\right|^{p}\right) \\
\leqslant & \left(2^{p}+1\right) \sum_{j=1}^{k}\left|\alpha_{n_{j}}\right|^{p} \leqslant\left(2^{p}+1\right)\|x\|_{\ell_{p}}^{p} .
\end{aligned}
$$

The result now follows by taking the supremum over all permissible sets $A$.

We have not been able to decide whether or not Proposition 4.12 is true for $p=1$.

Question 4.14. Is the standard basis $\left(e_{n}\right)_{n \in \mathbb{N}}$ for $V_{1}$ shrinking?

Note ADDED IN PROOF. Question 4.14 has been answered in the positive; see [10].

Lemma 4.15. Let $p \geqslant 1$. Then $W_{p}$ and $\mathbb{K} \chi_{\mathbb{N}}$ are closed, complementary subspaces of $\operatorname{bip}\left(V_{p}\right)$, and $\|w\|_{W_{p}}=\|w\|_{\operatorname{bip}\left(V_{p}\right)}$ for each $w \in W_{p}$.

Proof. We have $W_{p} \cap \mathbb{K}_{\mathbb{N}}=\{0\}$ because $W_{p} \subseteq c_{0}$ by definition.

Lemma 4.3 iii) implies that

$$
\|w\|_{\operatorname{bip}\left(V_{p}\right)} \leqslant\|w\|_{W_{p}} \quad\left(w \in W_{p}\right)
$$

so that in particular $W_{p} \subseteq \operatorname{bip}\left(V_{p}\right)$. Moreover, $\chi_{\mathbb{N}} \in \operatorname{bip}\left(V_{p}\right)$ because $P_{m} \chi_{\mathbb{N}}=\chi_{[1, m]}$ is a unit vector in $V_{p}$ for each $m \in \mathbb{N}$, and consequently $W_{p}+\mathbb{K} \chi_{\mathbb{N}} \subseteq \operatorname{bip}\left(V_{p}\right)$.

Conversely, suppose that $x \in \operatorname{bip}\left(V_{p}\right)$. We claim that the sequence $x=\left(\left\langle x, f_{n}\right\rangle\right)_{n \in \mathbb{N}}$ is convergent in $\mathbb{K}$. If not, $x$ fails to be a Cauchy sequence, so we can find $\varepsilon>0$ and integers $1=N_{1}<N_{2}<\cdots<N_{j}<N_{j+1}<\cdots$ such that $\left|\left\langle x, f_{N_{j}}-f_{N_{j+1}}\right\rangle\right| \geqslant \varepsilon$ for each $j \in \mathbb{N}$. Take $k \in \mathbb{N}$ such that $k \varepsilon^{p}>\|x\|_{\operatorname{bip}\left(V_{p}\right)}^{p}$, and choose $m \in \mathbb{N}$ such that $N_{m} \geqslant k$. The set $A:=\left\{N_{m}<N_{m+1}<\cdots<N_{m+k}\right\}$ is then permissible, and therefore we have

$$
\|x\|_{\mathrm{bip}\left(V_{p}\right)}^{p} \geqslant \nu_{p}\left(P_{N_{m+k}} x, A\right)^{p}=\sum_{j=m}^{m+k-1}\left|\left\langle x, f_{N_{j}}-f_{N_{j+1}}\right\rangle\right|^{p} \geqslant k \varepsilon^{p}>\|x\|_{\mathrm{bip}\left(V_{p}\right)}^{p}
$$

which is clearly absurd. Thus $x$ is convergent with limit $\alpha \in \mathbb{K}$, say. 
We now claim that $w:=x-\alpha \chi_{\mathbb{N}}$ belongs to $W_{p}$; indeed, $w \in c_{0}$ by the choice of $\alpha$, and

$$
\|w\|_{W_{p}} \leqslant\|x\|_{\operatorname{bip}\left(V_{p}\right)}
$$

because, for each permissible subset $A=\left\{n_{1}<n_{2}<\cdots<n_{k+1}\right\}$ of $\mathbb{N}$, we have

$$
\nu_{p}(w, A)^{p}=\sum_{j=1}^{k}\left|\left(\left\langle x, f_{n_{j}}\right\rangle-\alpha\right)-\left(\left\langle x, f_{n_{j+1}}\right\rangle-\alpha\right)\right|^{p}=\nu_{p}\left(P_{n_{k+1}} x, A\right)^{p} \leqslant\|x\|_{\mathrm{bip}\left(V_{p}\right)}^{p} .
$$

Hence we conclude that $x=w+\alpha \chi_{\mathbb{N}} \in W_{p}+\mathbb{K}_{\chi_{\mathbb{N}}}$, and therefore $\operatorname{bip}\left(V_{p}\right)=W_{p}+\mathbb{K} \chi_{\mathbb{N}}$.

To complete the proof, we note that if $x \in W_{p}$ in the argument given above, then $\alpha=0$, so that $w=x$, and therefore 4.5-4.6) combine to show that $\|w\|_{W_{p}}=\|w\|_{\operatorname{bip}\left(V_{p}\right)}$ for each $w \in W_{p}$. In particular, this implies that the subspace $W_{p}$ is closed in $\operatorname{bip}\left(V_{p}\right)$ because it is complete with respect to the norm $\|\cdot\|_{W_{p}}$ and thus also with respect to $\|\cdot\|_{\operatorname{bip}\left(V_{p}\right)}$. The subspace $\mathbb{K} \chi_{\mathbb{N}}$ is closed because it is finite-dimensional.

REMARK 4.16. Lemma 4.15 can be rephrased as follows: $\left(\operatorname{bip}\left(V_{p}\right),\|\cdot\|_{\operatorname{bip}\left(V_{p}\right)}\right)$ contains $\left(W_{p},\|\cdot\|_{W_{p}}\right)$ isometrically, and $\lambda:\left(\alpha_{n}\right)_{n \in \mathbb{N}} \mapsto \lim _{n \rightarrow \infty} \alpha_{n}$ defines a bounded functional on $\operatorname{bip}\left(V_{p}\right)$ whose kernel is $W_{p}$.

4.17. The unrestricted James-Schreier space and the bidual of $V_{p}$. For $p>1$, we can summarize our findings in the commutative diagram

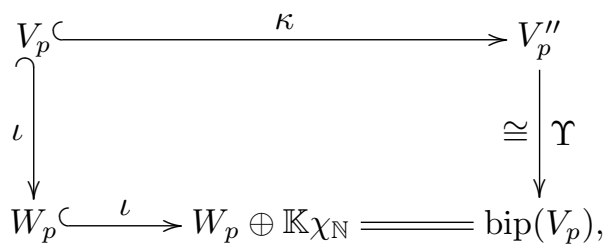

where both $\iota$ 's are the natural inclusions, $\kappa$ is the canonical embedding, and $\Upsilon$ is the isometric isomorphism from $\$ 2.4$ Thus, if we identify the bidual of $V_{p}$ with $\operatorname{bip}\left(V_{p}\right)$ via $\Upsilon$, then $\kappa=\iota$, that is, the canonical embedding of $V_{p}$ into its bidual becomes the natural inclusion of $V_{p}$ into $W_{p} \oplus \mathbb{K} \chi_{\mathbb{N}}$.

Finally in this section we take a look at shift operators on the James-Schreier spaces. We begin with an easy result that characterizes the increasing mappings $\sigma: \mathbb{N} \rightarrow \mathbb{N}$ for which the associated left and right shifts $\Lambda_{\sigma}$ and $R_{\sigma}$ (as defined in (3.2) ) are bounded as operators on $\left(c_{00},\|\cdot\|_{V_{p}}\right)$.

Proposition 4.18. Let $p \geqslant 1$, and let $\sigma: \mathbb{N} \rightarrow \mathbb{N}$ be strictly increasing. Then:

(i) $\left\|\Lambda_{\sigma} x\right\|_{V_{p}} \leqslant\|x\|_{V_{p}}$ for each $x \in c_{00}$, so that $\Lambda_{\sigma}$ extends uniquely to a contractive operator on $V_{p}$, no matter what $\sigma$ is;

(ii) the operator $R_{\sigma}$ is bounded on $\left(c_{00},\|\cdot\|_{V_{p}}\right)$ if and only if $\sigma(\mathbb{N})$ is cofinite in $\mathbb{N}$.

Proof. (ii). Given $x \in c_{00}$ and a permissible subset $A=\left\{n_{1}<\cdots<n_{k+1}\right\}$ of $\mathbb{N}$, we have

$$
\begin{aligned}
\nu_{p}\left(\Lambda_{\sigma} x, A\right)^{p}=\sum_{j=1}^{k}\left|\left\langle\Lambda_{\sigma} x, f_{n_{j}}-f_{n_{j+1}}\right\rangle\right|^{p} & =\sum_{j=1}^{k}\left|\left\langle x, f_{\sigma\left(n_{j}\right)}-f_{\sigma\left(n_{j+1}\right)}\right\rangle\right|^{p} \\
& =\nu_{p}(x, \sigma(A))^{p} \leqslant\|x\|_{V_{p}}^{p}
\end{aligned}
$$


where the final inequality follows from the fact that the set $\sigma(A)$ is permissible (because $\sigma$ is strictly increasing).

(ii). It is easy to see that $R_{\sigma}$ is bounded if $\sigma(\mathbb{N})$ is cofinite in $\mathbb{N}$.

Conversely, suppose that the set $\mathbb{N} \backslash \sigma(\mathbb{N})$ is infinite, and take a strictly increasing sequence $\left(m_{j}\right)_{j \in \mathbb{N}}$ of natural numbers such that $\sigma\left(m_{j}\right)+1 \notin \sigma(\mathbb{N})$ for each $j \in \mathbb{N}$. Then, for given $k \in \mathbb{N}$, we can find $n \in \mathbb{N}$ such that $\sigma\left(m_{n}\right) \geqslant 2 k+1$. This implies that the set $A:=\left\{\sigma\left(m_{j}\right), \sigma\left(m_{j}\right)+1: n \leqslant j \leqslant n+k\right\}$ is permissible, and therefore we have

$$
\left\|R_{\sigma} \chi_{\left[1, m_{n+k}\right]}\right\|_{V_{p}}^{p} \geqslant \nu_{p}\left(R_{\sigma} \chi_{\left[1, m_{n+k}\right]}, A\right)^{p}=2 k+1 .
$$

Since $\left\|\chi_{[1, m]}\right\|_{V_{p}}=1$, no matter what $m \in \mathbb{N}$ is, while $(2 k+1)^{\frac{1}{p}} \rightarrow \infty$ as $k \rightarrow \infty$, we conclude that the operator $R_{\sigma}$ is unbounded on $\left(c_{00},\|\cdot\|_{V_{p}}\right)$.

Thus, if we want to right-shift elements of $V_{p}$ in a non-trivial way, a different approach which does not introduce 'gaps' between coordinates is required. This is the motivation behind the following definition.

Definition 4.19. Let $\sigma: \mathbb{N} \rightarrow \mathbb{N}$ be strictly increasing. We associate with $\sigma$ the block right shift

$$
\Theta_{\sigma}: \sum_{n=1}^{\infty} \alpha_{n} e_{n} \mapsto \sum_{n=1}^{\infty} \alpha_{n} \chi_{(\sigma(n-1), \sigma(n)]}, \quad c_{00} \rightarrow c_{00},
$$

with the standing convention that $\sigma(0):=0$.

This block right shift is bounded on $\left(c_{00},\|\cdot\|_{V_{p}}\right)$ under exactly the same condition that the usual right shift is bounded on $\left(c_{00},\|\cdot\|_{S_{p}}\right)$ (see Corollary 3.17 ii ), as the following proposition shows; this result will be important in the study [9] of $V_{p}$ as a Banach algebra.

Proposition 4.20. Let $p \geqslant 1$, and let $\sigma: \mathbb{N} \rightarrow \mathbb{N}$ be strictly increasing. The block right shift $\Theta_{\sigma}$ is bounded on $\left(c_{00},\|\cdot\|_{V_{p}}\right)$ if and only if there is a constant $K \in \mathbb{N}$ such that $\sigma(n) \leqslant K n$ for each $n \in \mathbb{N}$. In the positive case, $\Theta_{\sigma}$ extends uniquely to a bounded operator of norm at most $K^{\frac{1}{p}}$ on $V_{p}$.

Proof. $\Leftarrow$. Suppose that there is a constant $K \in \mathbb{N}$ such that $\sigma(n) \leqslant K n$ for each $n \in \mathbb{N}$, and let $x \in c_{00}$ and a permissible subset $A$ of $\mathbb{N}$ be given. Take natural numbers $k$ and $m_{1}<m_{2}<\cdots<m_{k}$ and non-empty sets $A_{1}, \ldots, A_{k}$ with $A_{j} \subseteq\left(\sigma\left(m_{j}-1\right), \sigma\left(m_{j}\right)\right]$ for each $j \in\{1, \ldots, k\}$ (with the convention that $\sigma(0)=0$ ) such that $A=\bigcup_{j=1}^{k} A_{j}$. If $k=1$, then $\nu_{p}\left(\Theta_{\sigma} x, A\right)=0$, whereas for $k \geqslant 2$, we have

$$
\begin{aligned}
\nu_{p}\left(\Theta_{\sigma} x, A\right)^{p} & =\sum_{j=1}^{k-1}\left(\nu_{p}\left(\Theta_{\sigma} x, A_{j}\right)^{p}+\left|\left\langle\Theta_{\sigma} x, f_{\max A_{j}}-f_{\min A_{j+1}}\right\rangle\right|^{p}\right)+\nu_{p}\left(\Theta_{\sigma} x, A_{k}\right)^{p} \\
& =\sum_{j=1}^{k-1}\left|\left\langle\Theta_{\sigma} x, f_{\max A_{j}}-f_{\min A_{j+1}}\right\rangle\right|^{p}=\sum_{j=1}^{k-1}\left|\left\langle x, f_{m_{j}}-f_{m_{j+1}}\right\rangle\right|^{p}
\end{aligned}
$$

The permissibility of $A$ and our assumption on $\sigma$ imply that

$$
k \leqslant \operatorname{card} A \leqslant 1+\min A \leqslant 1+\sigma\left(m_{1}\right) \leqslant 1+K m_{1} .
$$

If this inequality is strict, choose integers $m_{1+K m_{1}}>m_{K m_{1}}>\cdots>m_{k+1}$, all greater than $m_{k}$. We can then define $B_{j}:=\left\{m_{h}:(j-1) m_{1}+1 \leqslant h \leqslant j m_{1}+1\right\} \subseteq \mathbb{N}$ for each 
$j=\{1, \ldots, K\}$, and this set is permissible because card $B_{j}=1+m_{1} \leqslant 1+\min B_{j}$. Hence we conclude that

$$
K\|x\|_{V_{p}}^{p} \geqslant \sum_{j=1}^{K} \nu_{p}\left(x, B_{j}\right)^{p}=\sum_{j=1}^{K m_{1}}\left|\left\langle x, f_{m_{j}}-f_{m_{j+1}}\right\rangle\right|^{p} \geqslant \nu_{p}\left(\Theta_{\sigma} x, A\right)^{p},
$$

where the final inequality follows from $4.8-(4.9)$. Taking the supremum over all permissible sets $A$, we obtain $K\|x\|_{V_{p}}^{p} \geqslant\left\|\Theta_{\sigma} x\right\|_{V_{p}}^{p}$, so that $\Theta_{\sigma}$ is bounded with norm at most $K^{\frac{1}{p}}$.

$\Rightarrow$. Conversely, suppose that $\Theta_{\sigma}$ is bounded on $\left(c_{00},\|\cdot\|_{V_{p}}\right)$, and consider the strictly increasing mappings $\gamma, \tau: \mathbb{N} \rightarrow \mathbb{N}$ given by $\gamma(n):=2 n+1$ and $\tau(n):=\sigma(2 n-1)+1$ for each $n \in \mathbb{N}$. We then see that the diagram

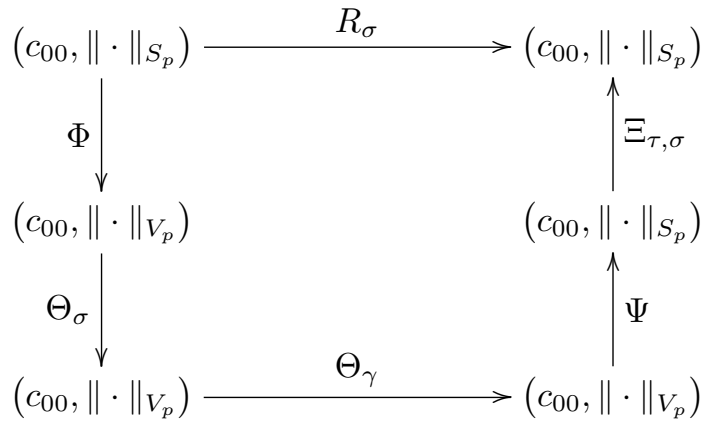

is commutative because

$$
\begin{aligned}
\Xi_{\tau, \sigma} \Psi \Theta_{\gamma} \Theta_{\sigma} \Phi e_{n} & =\Xi_{\tau, \sigma} \Psi \Theta_{\gamma} \Theta_{\sigma} e_{2 n}=\Xi_{\tau, \sigma} \Psi \Theta_{\gamma} \chi_{(\sigma(2 n-1), \sigma(2 n)]} \\
& =\Xi_{\tau, \sigma} \Psi \chi_{(2 \sigma(2 n-1)+1,2 \sigma(2 n)+1]}=\Xi_{\tau, \sigma}\left(e_{\sigma(2 n-1)+1}-e_{\sigma(2 n)+1}\right) \\
& =\Xi_{\tau, \sigma} e_{\tau(n)}-\Xi_{\tau, \sigma} e_{\sigma(2 n)+1}=e_{\sigma(n)}-0=R_{\sigma} e_{n} \quad(n \in \mathbb{N}) .
\end{aligned}
$$

Moreover, the operators $\Phi, \Theta_{\sigma}, \Theta_{\gamma}, \Psi$, and $\Xi_{\tau, \sigma}$ are all bounded with respect to the norms on their domains and codomains specified in the diagram; this follows from Proposition 4.10 for $\Phi$ and $\Psi$, from our assumption for $\Theta_{\sigma}$, from the implication ' $\Leftarrow$ ' proved above for $\Theta_{\gamma}$ (because $\gamma(n)=2 n+1 \leqslant 3 n$ for each $\left.n \in \mathbb{N}\right)$, and from Lemma 3.16 for $\Xi_{\tau, \sigma}$ (because $\sigma(n) \leqslant \sigma(2 n-1)+1=\tau(n)$ for each $n \in \mathbb{N}$ ). Hence the commutativity of the diagram implies that $R_{\sigma}$ is bounded, and therefore we have $\sigma(n) \leqslant K n$ for each $n \in \mathbb{N}$ by Corollary 3.17 ii .

Recall that the $p^{\text {th }}$ James space $J_{p}$ is not isomorphic to its Cartesian square for any $p>1$, whereas we saw in Corollary 3.19 that the $p^{\text {th }}$ Schreier space $S_{p}$ is isomorphic to its Cartesian square for each $p \geqslant 1$. We have not been able to answer the corresponding question for the James-Schreier spaces.

QUeSTION 4.21. Let $p \geqslant 1$. Is the $p^{\text {th }}$ James-Schreier space $V_{p}$ isomorphic to its Cartesian square $V_{p} \oplus V_{p}$ ?

\section{The James-Schreier spaces are $c_{0}$-saturated}

Definition 5.1. Let $X$ and $Y$ be infinite-dimensional Banach spaces. We say that $X$ is $Y$-saturated if each closed, infinite-dimensional subspace of $X$ contains a subspace which is isomorphic to $Y$. 
The aim of this section is to prove the following result.

TheOREm 5.2. For each $p \geqslant 1$, the $p^{\text {th }}$ James-Schreier space $V_{p}$ is $c_{0}$-saturated.

We isolate the key step in the proof of Theorem 5.2 in the following lemma.

Lemma 5.3. Let $p \geqslant 1$. Every block basic sequence of the standard basis for $V_{p}$ has a block basic sequence which is equivalent to the standard unit vector basis for $c_{0}$.

Proof. Suppose that $\left(u_{n}\right)_{n \in \mathbb{N}}$ is a block basic sequence of $\left(e_{n}\right)_{n \in \mathbb{N}}$, and choose integers $0=L_{0}<L_{1}<L_{2}<\cdots$ such that $u_{n} \in \operatorname{span}\left\{e_{j}: L_{n-1}<j \leqslant L_{n}\right\}$ for each $n \in \mathbb{N}$. By induction, we shall construct sequences $\left(M_{n}\right)_{n \in \mathbb{N}}$ and $\left(N_{n}\right)_{n \in \mathbb{N}}$ of natural numbers with $M_{1} \leqslant N_{1}<M_{2} \leqslant N_{2}<\cdots$ and a sequence $\left(v_{n}\right)_{n \in \mathbb{N}}$ of unit vectors in $V_{p}$ such that

$$
v_{n} \in \operatorname{span}\left\{u_{j}: M_{n} \leqslant j \leqslant N_{n}\right\} \quad \text { and } \quad\left|\left\langle v_{n+1}, f_{k}\right\rangle\right| \leqslant L_{N_{n}}^{-\frac{1}{p}} \quad(n, k \in \mathbb{N}) .
$$

To start the induction, take $M_{1}:=N_{1}:=1$ and $v_{1}:=u_{1} /\left\|u_{1}\right\|_{V_{p}} \in V_{p}$. Then $v_{1}$ is a unit vector which satisfies the first part of (5.1) by definition; the second part is void in this case.

Now let $n \in \mathbb{N}$, and assume that natural numbers $M_{1} \leqslant N_{1}<\cdots<M_{n} \leqslant N_{n}$ and unit vectors $v_{1}, \ldots, v_{n}$ in $V_{p}$ have been chosen in accordance with (5.1).

If there is an integer $m>N_{n}$ such that $\left|\left\langle u_{m}, f_{k}\right\rangle\right| \leqslant L_{N_{n}}^{-\frac{1}{p}}\left\|u_{m}\right\|_{V_{p}}$ for each $k \in \mathbb{N}$, then we can simply take $M_{n+1}:=N_{n+1}:=m$ and $v_{n+1}:=u_{m} /\left\|u_{m}\right\|_{V_{p}} \in V_{p}$.

Otherwise, for each $m>N_{n}$, we can find $k_{m} \in \mathbb{N}$ such that

$$
\left|\left\langle u_{m}, f_{k_{m}}\right\rangle\right|>L_{N_{n}}^{-\frac{1}{p}}\left\|u_{m}\right\|_{V_{p}} .
$$

In particular, $\left\langle u_{m}, f_{k_{m}}\right\rangle \neq 0$, so that

$$
L_{m-1}<k_{m} \leqslant L_{m},
$$

and therefore the sequence $\left(k_{m}\right)_{m=N_{n}+1}^{\infty}$ is strictly increasing. We now choose an integer $K \geqslant 2^{-p}\left(L_{N_{n}}^{2}-1\right)+1$, we then pick an integer $M_{n+1}>N_{n}$ such that $k_{M_{n+1}} \geqslant K$, and finally we define $N_{n+1}:=M_{n+1}+K-1 \geqslant M_{n+1}$. These choices ensure that the set $A:=\left\{k_{m}: M_{n+1} \leqslant m \leqslant N_{n+1}+1\right\}$ is permissible, and consequently we can estimate the James-Schreier norm of the vector

$$
w:=\sum_{j=M_{n+1}}^{N_{n+1}} \alpha_{j} u_{j} \in V_{p}, \quad \text { where } \quad \alpha_{j}:=\frac{(-1)^{j} \overline{\left\langle u_{j}, f_{k_{j}}\right\rangle}}{\left|\left\langle u_{j}, f_{k_{j}}\right\rangle\right| \cdot\left\|u_{j}\right\|_{V_{p}}} \in \mathbb{K},
$$

as follows:

$$
\begin{aligned}
\|w\|_{V_{p}}^{p} & \geqslant \nu_{p}(w, A)^{p}=\sum_{m=M_{n+1}}^{N_{n+1}}\left|\sum_{j=M_{n+1}}^{N_{n+1}} \alpha_{j}\left(\left\langle u_{j}, f_{k_{m}}\right\rangle-\left\langle u_{j}, f_{k_{m+1}}\right\rangle\right)\right|^{p} \\
\text { by } & =\sum_{m=M_{n+1}}^{N_{n+1}-1}\left|\alpha_{m}\left\langle u_{m}, f_{k_{m}}\right\rangle-\alpha_{m+1}\left\langle u_{m+1}, f_{k_{m+1}}\right\rangle\right|^{p}+\left|\alpha_{N_{n+1}}\left\langle u_{N_{n+1}}, f_{k_{N_{n+1}}}\right\rangle\right|^{p}
\end{aligned}
$$




$$
\begin{aligned}
& \text { by } \underset{\overline{\overline{5}}}{\overline{5.4}} \sum_{m=M_{n+1}}^{N_{n+1}-1}\left(\frac{\left|\left\langle u_{m}, f_{k_{m}}\right\rangle\right|}{\left\|u_{m}\right\|_{V_{p}}}+\frac{\left|\left\langle u_{m+1}, f_{k_{m+1}}\right\rangle\right|}{\left\|u_{m+1}\right\|_{V_{p}}}\right)^{p}+\left(\frac{\left|\left\langle u_{N_{n+1}}, f_{k_{N_{n+1}}}\right\rangle\right|}{\left\|u_{N_{n+1}}\right\|_{V_{p}}}\right)^{p} \\
& \text { by } \underset{5.2}{>} \sum_{m=M_{n+1}}^{N_{n+1}-1}\left(2 L_{N_{n}}^{-\frac{1}{p}}\right)^{p}+\left(L_{N_{n}}^{-\frac{1}{p}}\right)^{p}=\frac{2^{p}(K-1)+1}{L_{N_{n}}} \geqslant L_{N_{n}}
\end{aligned}
$$

by the choice of $K$. In particular we have $w \neq 0$, so $v_{n+1}:=w /\|w\|_{V_{p}}$ defines a unit vector in $V_{p}$, and (5.4) implies that this vector satisfies the first part of (5.1). To verify the second part, we use the fact that $\left(u_{j}\right)_{j \in \mathbb{N}}$ is a block basic sequence to obtain

$$
\begin{aligned}
\left|\left\langle v_{n+1}, f_{k}\right\rangle\right| & =\frac{1}{\|w\|_{V_{p}}} \max \left\{\left|\alpha_{j}\left\langle u_{j}, f_{k}\right\rangle\right|: M_{n+1} \leqslant j \leqslant N_{n+1}\right\} \\
& =\frac{1}{\|w\|_{V_{p}}} \max \left\{\frac{\left|\left\langle u_{j}, f_{k}\right\rangle\right|}{\left\|u_{j}\right\|_{V_{p}}}: M_{n+1} \leqslant j \leqslant N_{n+1}\right\} \leqslant \frac{1}{\|w\|_{V_{p}}} \leqslant L_{N_{n}}^{-\frac{1}{p}} \quad(k \in \mathbb{N}),
\end{aligned}
$$

where the final inequality follows from $[5.5$. Hence the induction continues.

We shall next show that the block basic sequence $\left(v_{n}\right)_{n \in \mathbb{N}}$ constructed above is equivalent to the standard unit vector basis of $c_{0}$; this will clearly complete the proof. To this end, we consider the linear mapping

$$
U: \sum_{n=1}^{\infty} \beta_{n} e_{n} \mapsto \sum_{n=1}^{\infty} \beta_{n} v_{n}, \quad\left(c_{00},\|\cdot\|_{c_{0}}\right) \rightarrow V_{p} .
$$

Our aim is to show that $U$ is an isomorphism onto its image, that is, we want to prove that there are constants $C_{1}, C_{2}>0$ such that

$$
C_{1}\|x\|_{c_{0}} \leqslant\|U x\|_{V_{p}} \leqslant C_{2}\|x\|_{c_{0}} \quad\left(x \in c_{00}\right) .
$$

Given $x=\sum_{n=1}^{\infty} \beta_{n} e_{n} \in c_{00}$, take $\ell \in \mathbb{N}$ such that $\|x\|_{c_{0}}=\left|\beta_{\ell}\right|$. Then we have

$$
\begin{aligned}
\|x\|_{c_{0}} & =\left\|\beta_{\ell} v_{\ell}\right\|_{V_{p}} \\
& =\left\{\begin{array}{ll}
\left\|P_{L_{N_{1}}}(U x)\right\|_{V_{p}} \leqslant\left\|P_{L_{N_{1}}}\right\|\|U x\|_{V_{p}} & \text { for } \ell=1 \\
\left\|\left(P_{L_{N_{\ell}}}-P_{L_{N_{\ell-1}}}\right) U x\right\|_{V_{p}} \leqslant\left\|P_{L_{N_{\ell}}}-P_{L_{N_{\ell-1}}}\right\|\|U x\|_{V_{p}} & \text { for } \ell \geqslant 2
\end{array}\right\} \leqslant 2\|U x\|_{V_{p}}
\end{aligned}
$$

by Lemma 4.3 ii). Hence the first inequality in (5.6) holds with $C_{1}:=1 / 2$.

To prove the second, let a permissible subset $A=\left\{n_{1}<n_{2}<\cdots<n_{k+1}\right\}$ of $\mathbb{N}$ be given. Choose $h \in \mathbb{N}$ minimal such that $n_{1} \leqslant L_{N_{h}}$, and then take $g \in\{1, \ldots, k+1\}$ maximal such that $n_{g} \leqslant L_{N_{h}}$. If $g=k+1$, then $A \subseteq\left(L_{N_{h-1}}, L_{N_{h}}\right]$ (with $N_{0}:=0$ if $h=1$ ), and we have

$$
\nu_{p}(U x, A)^{p}=\nu_{p}\left(\beta_{h} v_{h}, A\right)^{p} \leqslant\left\|\beta_{h} v_{h}\right\|_{V_{p}}^{p}=\left|\beta_{h}\right|^{p} \leqslant\|x\|_{c_{0}}^{p} .
$$

Otherwise (that is, when $g \leqslant k$ ) we write

$$
\nu_{p}(U x, A)^{p}=\sum_{j=1}^{g-1}\left|\left\langle U x, f_{n_{j}}-f_{n_{j+1}}\right\rangle\right|^{p}+\left|\left\langle U x, f_{n_{g}}-f_{n_{g+1}}\right\rangle\right|^{p}+\sum_{j=g+1}^{k}\left|\left\langle U x, f_{n_{j}}-f_{n_{j+1}}\right\rangle\right|^{p},
$$

where we ignore the left-hand sum if $g=1$ and the right-hand sum if $g=k$. Application of the inequality (4.1) to the middle term and the right-hand sum (but not the left-hand 
sum) yields

$$
\begin{aligned}
& \nu_{p}(U x, A)^{p} \leqslant \sum_{j=1}^{g-1}\left|\left\langle U x, f_{n_{j}}-f_{n_{j+1}}\right\rangle\right|^{p} \\
&+2^{p-1}\left(\left|\left\langle U x, f_{n_{g}}\right\rangle\right|^{p}+\left|\left\langle U x, f_{n_{g+1}}\right\rangle\right|^{p}\right) \\
&+2^{p-1}\left(\sum_{j=g+1}^{k}\left|\left\langle U x, f_{n_{j}}\right\rangle\right|^{p}+\left|\left\langle U x, f_{n_{j+1}}\right\rangle\right|^{p}\right) \\
& \leqslant 2^{p-1}\left(\sum_{j=1}^{g-1}\left|\left\langle U x, f_{n_{j}}-f_{n_{j+1}}\right\rangle\right|^{p}+\left|\left\langle U x, f_{n_{g}}\right\rangle\right|^{p}\right)+2^{p} \sum_{j=g+1}^{k+1}\left|\left\langle U x, f_{n_{j}}\right\rangle\right|^{p} .
\end{aligned}
$$

We estimate the two terms on the right-hand side of this inequality separately. Firstly, the set $B:=\left\{n_{1}<n_{2}<\cdots<n_{g}<L_{N_{h}}+1\right\}$ is permissible because $g \leqslant k \leqslant n_{1}$, and therefore we have

$$
\sum_{j=1}^{g-1}\left|\left\langle U x, f_{n_{j}}-f_{n_{j+1}}\right\rangle\right|^{p}+\left|\left\langle U x, f_{n_{g}}\right\rangle\right|^{p}=\nu_{p}\left(\beta_{h} v_{h}, B\right)^{p} \leqslant\left\|\beta_{h} v_{h}\right\|_{V_{p}}^{p}=\left|\beta_{h}\right|^{p} \leqslant\|x\|_{c_{0}}^{p} .
$$

Secondly, we observe that

$$
\begin{aligned}
\sum_{j=g+1}^{k+1}\left|\left\langle U x, f_{n_{j}}\right\rangle\right|^{p} & \leqslant(k-g+1) \sup \left\{\left|\left\langle U x, f_{n}\right\rangle\right|^{p}: n \geqslant n_{g+1}\right\} \\
& \leqslant k \sup \left\{\left|\left\langle U x, f_{n}\right\rangle\right|^{p}: n>L_{N_{h}}\right\} \\
& =k \sup \left\{\left|\beta_{j}\right|^{p}\left|\left\langle v_{j}, f_{n}\right\rangle\right|^{p}: n>L_{N_{h}}, j>h\right\} \leqslant k\|x\|_{c_{0}}^{p}\left(L_{N_{h}}^{-\frac{1}{p}}\right)^{p} \leqslant\|x\|_{c_{0}}^{p}
\end{aligned}
$$

because $k \leqslant n_{1} \leqslant L_{N_{h}}$. Hence we conclude that

$$
\nu_{p}(U x, A)^{p} \leqslant 2^{p-1}\|x\|_{c_{0}}^{p}+2^{p}\|x\|_{c_{0}}^{p}=3 \cdot 2^{p-1}\|x\|_{c_{0}}^{p},
$$

and this, together with (5.7), shows that the second inequality in $(5.6)$ is satisfied with $C_{2}:=\left(3 \cdot 2^{p-1}\right)^{\frac{1}{p}}=3^{\frac{1}{p}} \cdot 2^{1-\frac{1}{p}}$.

Proof of Theorem 5.2. Let $X$ be a closed, infinite-dimensional subspace of $V_{p}$. By a theorem of Bessaga and Pełczyński (see [7], or [27, Proposition 1.a.11] for an exposition), $X$ contains a sequence $\left(x_{n}\right)_{n \in \mathbb{N}}$ which is equivalent to a block basic sequence $\left(u_{n}\right)_{n \in \mathbb{N}}$ of the standard basis for $V_{p}$. Lemma 5.3 implies that $\left(u_{n}\right)_{n \in \mathbb{N}}$ has a block basic sequence $\left(v_{n}\right)_{n \in \mathbb{N}}$ which is equivalent to the standard unit vector basis for $c_{0}$. Since $\left(u_{n}\right)_{n \in \mathbb{N}}$ is equivalent to $\left(x_{n}\right)_{n \in \mathbb{N}},\left(v_{n}\right)_{n \in \mathbb{N}}$ is equivalent to a block basic sequence of $\left(x_{n}\right)_{n \in \mathbb{N}}$, say $\left(y_{n}\right)_{n \in \mathbb{N}}$, and consequently we have

$$
X \supseteq \overline{\operatorname{span}}\left\{x_{n}: n \in \mathbb{N}\right\} \supseteq \overline{\operatorname{span}}\left\{y_{n}: n \in \mathbb{N}\right\} \cong \overline{\operatorname{span}}\left\{v_{n}: n \in \mathbb{N}\right\} \cong c_{0},
$$

as desired.

Corollary 5.4. For each $p \geqslant 1$, the $p^{\text {th }}$ Schreier space $S_{p}$ is $c_{0}$-saturated.

Proof. This is immediate from the fact that $V_{p}$ contains a subspace isomorphic to $S_{p}$ (see Proposition 4.10. 
REMARK 5.5. (i) Corollary 5.4 is known in the case $p=1$ (e.g., see [15]).

(ii) Proposition 2.3, which is the counterpart of Theorem 5.2 and Corollary 5.4 for James spaces, appears to be formally stronger as it states that every closed, infinitedimensional subspace of $J_{p}$ contains a complemented subspace which is isomorphic to $\ell_{p}$; however, Sobczyk's Theorem (e.g., see [27, Theorem 2.f.5]) implies that every subspace of $V_{p}$ or $S_{p}$ (or any other separable Banach space) which is isomorphic to $c_{0}$ is complemented.

(iii) Corollary 5.4 implies that $\ell_{1}$ does not embed in $S_{p}$ for each $p \geqslant 1$. Since $\left(e_{n}\right)_{n \in \mathbb{N}}$ is an unconditional basis for $S_{p}$, a well-known theorem of James [22] shows that $\left(e_{n}\right)_{n \in \mathbb{N}}$ is shrinking, thus giving an alternative proof of Proposition 3.10 and Corollary 3.12 .

COROLlaRY 5.6. For each $p \geqslant 1$, the $p^{\text {th }}$ unrestricted Schreier space $Z_{p}$ contains a subspace which is isomorphic to $\ell_{\infty}$; the same is true for the $p^{\text {th }}$ unrestricted JamesSchreier space $W_{p}$.

Proof. Since $S_{p}$ contains a subspace which is isomorphic to $c_{0}$, its bidual $S_{p}^{\prime \prime}$ contains a subspace which is isomorphic to $c_{0}^{\prime \prime} \cong \ell_{\infty}$; this proves the first clause because $Z_{p} \cong S_{p}^{\prime \prime}$ by 3.14 . The second follows immediately from this because Proposition 4.5 implies that $W_{p}$ contains a subspace which is isomorphic to $Z_{p}$.

Definition 5.7. Two infinite-dimensional Banach spaces $X$ and $Y$ are totally incomparable if no closed, infinite-dimensional subspace of $X$ is isomorphic to a subspace of $Y$.

Any two distinct spaces from the family $\left\{\ell_{p}: 1 \leqslant p<\infty\right\} \cup\left\{c_{0}\right\}$ are totally incomparable, as is well-known (e.g., see [27, p. 54]).

Lemma 5.8. Let $X_{1}, X_{2}, Y_{1}$, and $Y_{2}$ be infinite-dimensional Banach spaces, and suppose that $X_{j}$ is $Y_{j}$-saturated for $j=1,2$ and that $Y_{1}$ and $Y_{2}$ are totally incomparable. Then $X_{1}$ and $X_{2}$ are totally incomparable.

Proof. Assume towards a contradiction that $E$ is a closed, infinite-dimensional subspace of $X_{1}$ which is isomorphic to a subspace of $X_{2}$. Then $E$ contains a subspace $F$ which is isomorphic to $Y_{1}$. Since $F$ is isomorphic to a subspace of $X_{2}$, it contains a subspace which is isomorphic to $Y_{2}$, contradicting that $Y_{1}$ and $Y_{2}$ are totally incomparable.

Corollary 5.9. Let $p, q \geqslant 1$. Then:

(i) $\ell_{p}$ and $S_{q}$ are totally incomparable;

(ii) $\ell_{p}$ and $V_{q}$ are totally incomparable;

(iii) $J_{p}$ and $S_{q}$ are totally incomparable;

(iv) $J_{p}$ and $V_{q}$ are totally incomparable.

Proof. We apply Lemma 5.8 with $X_{1}:=\ell_{p}$ or $X_{1}:=J_{p}, X_{2}:=S_{q}$ or $X_{2}:=V_{q}$, and $Y_{1}:=\ell_{p}$ and $Y_{2}:=c_{0}$. As mentioned above, $Y_{1}$ and $Y_{2}$ are totally incomparable, while [27, Proposition 2.a.2], Proposition 2.3. Corollary 5.4, and Theorem 5.2 imply that $X_{j}$ is $Y_{j}$-saturated for $j=1,2$.

Evidence from other sequence spaces suggests that no two Schreier spaces with distinct indices should be isomorphic and, likewise, no two James-Schreier spaces with distinct 
indices should be isomorphic. We have, unfortunately, been unable to verify any of these conjectures.

Question 5.10. Is it true that $S_{p} ¥ S_{q}$ and $V_{p} \nsucceq V_{q}$ whenever $1 \leqslant p<q<\infty$ ?

Note ADDED IN PROOF. Question 5.10 has been answered in the positive; see [10].

\section{The $p^{\text {th }}$ James-Schreier space does not embed in a Banach space with an} unconditional basis for $\boldsymbol{p}>\mathbf{1}$. The aim of this section is to prove the result stated in the title; in particular, this will imply that, for $p>1$, the $p^{\text {th }}$ James-Schreier space does not embed in any of the Schreier spaces.

Our proof relies on the following technical notion which was originally introduced by Pełczyński [32] who, motivated by work of Orlicz, was studying the relationship between weak completeness and weak unconditional convergence of series in Banach spaces.

Definition 6.1. A Banach space $X$ has Pełczyński's property (u) if, for every weak Cauchy sequence $\left(x_{n}\right)_{n \in \mathbb{N}}$ in $X$, there is a sequence $\left(y_{n}\right)_{n \in \mathbb{N}}$ in $X$ such that

$$
\sum_{n=1}^{\infty}\left|\left\langle y_{n}, f\right\rangle\right|<\infty \quad \text { and } \quad\left\langle x_{n}-\sum_{j=1}^{n} y_{j}, f\right\rangle \rightarrow 0 \quad \text { as } \quad n \rightarrow \infty \quad\left(f \in X^{\prime}\right) .
$$

The reason that this notion is relevant for our purposes is immediately explained by parts (i) and (ii) of the following result, which was stated in [32.

Theorem 6.2 (Pełczyński).

(i) Every Banach space with an unconditional basis has Pełczyński's property (u).

(ii) Every closed subspace of a Banach space with Petczyński's property (u) has Pełczyński's property $(\mathrm{u})$.

(iii) The James space $J_{2}$ does not have Pełczyński's property $(\mathrm{u})$.

Detailed proofs of all three parts of Theorem 6.2 are given in [1, Section 3.5]. A more general statement than Theorem 6.2 (i) can be found in [28, Proposition 1.c.2], namely: every order continuous Banach lattice has Pełczyński’s property (u).

We can now state the main result of this section, which is the counterpart of Theorem 6.2 iii) for James-Schreier spaces.

THEOREM 6.3. For each $p>1$, the $p^{\text {th }}$ James-Schreier space $V_{p}$ does not have Petczynski's property $(\mathrm{u})$.

The proof of Theorem 6.3 goes via two lemmas. The first is easy, so we omit its proof. LEMMA 6.4. Let $X$ be a Banach space with a shrinking basis $\left(b_{n}\right)_{n \in \mathbb{N}}$, and let $\left(b_{m}^{\prime}\right)_{m \in \mathbb{N}}$ be the associated biorthogonal functionals. A sequence $\left(x_{n}\right)_{n \in \mathbb{N}}$ in $X$ is a weak Cauchy sequence if and only if $\left(x_{n}\right)_{n \in \mathbb{N}}$ is bounded and the sequence $\left(\left\langle x_{n}, b_{m}^{\prime}\right\rangle\right)_{n \in \mathbb{N}}$ converges for each $m \in \mathbb{N}$.

LEMMA 6.5. Let $p \geqslant 1$, and suppose that $\left(y_{n}\right)_{n \in \mathbb{N}}$ is a sequence in $V_{p}$ such that

$$
\left\langle\chi_{[1, n]}-\sum_{j=1}^{n} y_{j}, f_{m}\right\rangle \rightarrow 0 \quad \text { as } n \rightarrow \infty \quad(m \in \mathbb{N}) .
$$

Then the series $\sum_{n=1}^{\infty}\left|\left\langle y_{n}, f\right\rangle\right|$ diverges for some $f \in V_{p}^{\prime}$. 
Before we engage with the proof of Lemma 6.5. let us see how these two lemmas combine to establish our main result.

Proof of Theorem 6.3. Assume towards a contradiction that $V_{p}$ has Pełczyński's property $(\mathrm{u})$, and let $x_{n}:=\chi_{[1, n]}$ for each $n \in \mathbb{N}$. Since $p>1$, the standard basis for $V_{p}$ is shrinking, so Lemma 6.4 implies that $\left(x_{n}\right)_{n \in \mathbb{N}}$ is a weak Cauchy sequence in $V_{p}$. Therefore, by assumption, we can choose a sequence $\left(y_{n}\right)_{n \in \mathbb{N}}$ in $V_{p}$ which satisfies 6.1. This, however, contradicts Lemma 6.5 .

REMARK 6.6. Our proof of Theorem 6.3. like that of Theorem 6.2 iii) given in [1, starts by taking $x_{n}:=\chi_{[1, n]}$ and then proceeds by arguing that there is no sequence $\left(y_{n}\right)_{n \in \mathbb{N}}$ which satisfies 6.1. This follows quite easily for $J_{2}$ from general structure theorems because no subspace of $J_{2}$ is isomorphic to $c_{0}$. As we saw in Section 5 , this is no longer true for $V_{p}$, and so a different and, as it turns out, more delicate argument is required.

The following family of functionals will play a key role in the proof of Lemma 6.5.

Definition 6.7. Given a strictly increasing mapping $\sigma: \mathbb{N} \rightarrow \mathbb{N}$, we associate with it the alternating harmonic functional

$$
\xi_{\sigma}: x \mapsto \sum_{n=1}^{\infty} \frac{(-1)^{n}}{n}\left\langle x, f_{\sigma(n)}\right\rangle, \quad c_{00} \rightarrow \mathbb{K} .
$$

As one might guess, our interest in $\xi_{\sigma}$ stems from the fact that it extends to a bounded functional on $V_{p}$. This can be conveniently proved using the following lemma which, roughly speaking, states that in order to establish the boundedness of a functional on $V_{p}$, it suffices to consider its action on finite, positive vectors. To make this statement precise, we introduce the notation $c_{00}^{+}$for the positive cone of $c_{00}$, that is,

$$
c_{00}^{+}:=\left\{\sum_{j=1}^{n} \alpha_{j} e_{j}: n \in \mathbb{N}, \alpha_{1}, \ldots, \alpha_{n} \in[0, \infty)\right\} .
$$

LEMMA 6.8. Let $p \geqslant 1$, let $f$ be a functional on $c_{00}$, and suppose that there is a constant $C \geqslant 0$ such that $|\langle x, f\rangle| \leqslant C\|x\|_{V_{p}}$ for each $x \in c_{00}^{+}$. Then $f$ extends uniquely to a bounded functional on $V_{p}$, and the extension has norm at most $4 C$.

Proof. Given $x \in c_{00}$, let $x^{+}:=\left(\max \left\{\operatorname{Re}\left\langle x, f_{n}\right\rangle, 0\right\}\right)_{n \in \mathbb{N}} \in c_{00}^{+}$. Then, for each permissible subset $A=\left\{n_{1}<n_{2}<\cdots<n_{k+1}\right\}$ of $\mathbb{N}$, we have

$$
\begin{aligned}
\|x\|_{V_{p}}^{p} & \geqslant \sum_{j=1}^{k}\left|\left\langle x, f_{n_{j}}-f_{n_{j+1}}\right\rangle\right|^{p} \geqslant \sum_{j=1}^{k}\left|\operatorname{Re}\left\langle x, f_{n_{j}}\right\rangle-\operatorname{Re}\left\langle x, f_{n_{j+1}}\right\rangle\right|^{p} \\
& \geqslant \sum_{j=1}^{k}\left|\max \left\{\operatorname{Re}\left\langle x, f_{n_{j}}\right\rangle, 0\right\}-\max \left\{\operatorname{Re}\left\langle x, f_{n_{j+1}}\right\rangle, 0\right\}\right|^{p}=\nu_{p}\left(x^{+}, A\right)^{p},
\end{aligned}
$$

where the final inequality is an immediate consequence of the elementary estimate

$$
|\alpha-\beta| \geqslant|\max \{\alpha, 0\}-\max \{\beta, 0\}| \quad(\alpha, \beta \in \mathbb{R}) .
$$

Taking the supremum over all permissible sets $A$ in 6.4 , we obtain $\|x\|_{V_{p}} \geqslant\left\|x^{+}\right\|_{V_{p}}$.

Now define $x^{-}:=(-x)^{+}, x^{\mathrm{i}+}:=(-\mathrm{i} x)^{+}$, and $x^{\mathrm{i}-}:=(\mathrm{i} x)^{+}$. (Strictly speaking, the latter two definitions make sense only if the scalar field is $\mathbb{C}$; if $\mathbb{K}=\mathbb{R}$, let $x^{\mathrm{i} \pm}:=0$, no 
matter what $x$ is.) Then we have $x=x^{+}-x^{-}+\mathrm{i}\left(x^{\mathrm{i}+}-x^{\mathrm{i}-}\right)$, where $x^{ \pm}, x^{\mathrm{i} \pm} \in c_{00}^{+}$, and each of them has James-Schreier norm at most $\|x\|_{V_{p}}$ by the first part of the proof. Thus the assumption on $f$ implies that

$$
|\langle x, f\rangle| \leqslant\left|\left\langle x^{+}, f\right\rangle\right|+\left|\left\langle x^{-}, f\right\rangle\right|+\left|\left\langle x^{\mathrm{i}+}, f\right\rangle\right|+\left|\left\langle x^{\mathrm{i}-}, f\right\rangle\right| \leqslant 4 C\|x\|_{V_{p}},
$$

and the result follows from the density of $c_{00}$ in $V_{p}$.

Lemma 6.9. For each $p \geqslant 1$ and each strictly increasing mapping $\sigma: \mathbb{N} \rightarrow \mathbb{N}$, the functional $\xi_{\sigma}$ given by 6.3 extends uniquely to a bounded functional on $V_{p}$ of norm at most $4 \cdot 2^{\frac{1}{p}}\left(2^{\frac{1}{p}}-1\right)^{-1}$.

Proof. We begin with an elementary observation for later reference:

$$
\sum_{j=1}^{n}\left|\alpha_{j}\right| \leqslant n^{1-\frac{1}{p}}\left(\sum_{j=1}^{n}\left|\alpha_{j}\right|^{p}\right)^{\frac{1}{p}} \quad\left(n \in \mathbb{N}, \alpha_{1}, \ldots, \alpha_{n} \in \mathbb{K}\right) ;
$$

indeed, this inequality is trivial for $p=1$, whereas for $p>1$, application of Hölder's inequality to the $n$-tuples $(1,1, \ldots, 1)$ and $\left(\alpha_{1}, \alpha_{2}, \ldots, \alpha_{n}\right)$ gives the required estimate. We note in passing that 4.1 is a special case of 6.5 corresponding to $n=2$.

For each $m \in \mathbb{N}$, let $A_{m}:=\mathbb{N} \cap\left[2^{m}-1,2^{m+1}-2\right]$; this is a permissible set because card $A_{m}=2^{m}=\min A_{m}+1$, and therefore, as $\sigma$ is increasing, $\sigma\left(A_{m}\right)$ is also permissible. Now, for given $x \in c_{00}^{+}$, choose $N \in \mathbb{N}$ such that $\left\langle x, f_{\sigma(n)}\right\rangle=0$ whenever $n>2\left(2^{N}-1\right)$. Then we have

$$
\left\langle x, \xi_{\sigma}\right\rangle=\sum_{n=1}^{2\left(2^{N}-1\right)} \frac{(-1)^{n}}{n}\left\langle x, f_{\sigma(n)}\right\rangle=\sum_{n=1}^{2^{N}-1}\left(-\frac{\left\langle x, f_{\sigma(2 n-1)}\right\rangle}{2 n-1}+\frac{\left\langle x, f_{\sigma(2 n)}\right\rangle}{2 n}\right),
$$

which is real because $x \in c_{00}^{+}$; the following estimates yield an upper bound:

$$
\begin{aligned}
\left\langle x, \xi_{\sigma}\right\rangle & \leqslant \sum_{n=1}^{2^{N}-1} \frac{\left\langle x, f_{\sigma(2 n)}-f_{\sigma(2 n-1)}\right\rangle}{2 n} \leqslant \sum_{m=1}^{N}\left(\sum_{n=2^{m-1}}^{2^{m}-1} \frac{\left|\left\langle x, f_{\sigma(2 n-1)}-f_{\sigma(2 n)}\right\rangle\right|}{2^{m}}\right) \\
& \leqslant \sum_{m=1}^{N} \frac{1}{2^{m}} \sum_{j=2^{m}-1}^{2^{m+1}-3}\left|\left\langle x, f_{\sigma(j)}-f_{\sigma(j+1)}\right\rangle\right| \\
& \leqslant \sum_{m=1}^{N} \frac{\left(2^{m}-1\right)^{1-\frac{1}{p}}}{2^{m}}\left(\sum_{j=2^{m}-1}^{2^{m+1}-3}\left|\left\langle x, f_{\sigma(j)}-f_{\sigma(j+1)}\right\rangle\right|^{p}\right)^{\frac{1}{p}} \\
& =\sum_{m=1}^{N} \frac{\left(2^{m}-1\right)^{1-\frac{1}{p}}}{2^{m}} \nu_{p}\left(x, \sigma\left(A_{m}\right)\right) \leqslant \sum_{m=1}^{N}\left(2^{m}\right)^{-\frac{1}{p}}\|x\|_{V_{p}} \leqslant \frac{\|x\|_{V_{p}}}{2^{\frac{1}{p}}-1}
\end{aligned}
$$

by summation of the geometric progression.

On the other hand, pairing up neighbouring terms differently in 6.6, we obtain a lower bound: 


$$
\begin{aligned}
\left\langle x, \xi_{\sigma}\right\rangle & =-\left\langle x, f_{\sigma(1)}\right\rangle+\sum_{n=1}^{2^{N}-1}\left(\frac{\left\langle x, f_{\sigma(2 n)}\right\rangle}{2 n}-\frac{\left\langle x, f_{\sigma(2 n+1)}\right\rangle}{2 n+1}\right) \\
& \geqslant-\|x\|_{V_{p}}+\sum_{n=1}^{2^{N}-1} \frac{\left\langle x, f_{\sigma(2 n)}-f_{\sigma(2 n+1)}\right\rangle}{2 n} \geqslant-\frac{2^{\frac{1}{p}}\|x\|_{V_{p}}}{2^{\frac{1}{p}}-1},
\end{aligned}
$$

where the final inequality follows from estimates very similar to those used in the calculation above.

Combining the upper and lower bound, we see that $\left|\left\langle x, \xi_{\sigma}\right\rangle\right| \leqslant 2^{\frac{1}{p}}\left(2^{\frac{1}{p}}-1\right)^{-1}\|x\|_{V_{p}}$ for each $x \in c_{00}^{+}$, and hence Lemma 6.8 gives the result.

REMARK 6.10. It would suffice to prove Lemma 6.9 for $\sigma=\iota$ (the identity mapping on $\mathbb{N}$ ) because $\xi_{\sigma}=\Lambda_{\sigma}^{\prime} \xi_{\iota}$, where $\Lambda_{\sigma}$ is the left shift defined in (3.2). The proof of the boundedness of $\xi_{\iota}$ is not, however, significantly simpler than the general proof given above.

We are now ready to prove Lemma 6.5

Proof of Lemma 6.5. We split in two cases. Suppose first that, for some $k \in \mathbb{N}$ and $\varepsilon>0$, we have $\sum_{j=n}^{\infty}\left|\left\langle y_{j}, f_{k}\right\rangle\right|>\varepsilon$ for each $n \in \mathbb{N}$. Then the series $\sum_{j=1}^{\infty}\left|\left\langle y_{j}, f_{k}\right\rangle\right|$ diverges, so in this case we can simply take $f:=f_{k} \in V_{p}^{\prime}$.

Otherwise, for each $k \in \mathbb{N}$ and $\varepsilon>0$, we can find $n \in \mathbb{N}$ such that

$$
\sum_{j=n}^{\infty}\left|\left\langle y_{j}, f_{k}\right\rangle\right| \leqslant \varepsilon
$$

In this case our strategy is to construct a strictly increasing mapping $\sigma: \mathbb{N} \rightarrow \mathbb{N}$ such that the series $\sum_{n=1}^{\infty}\left|\left\langle y_{n}, \xi_{\sigma}\right\rangle\right|$ diverges. Fix a summable, decreasing sequence $\left(\varepsilon_{m}\right)_{m \in \mathbb{N}}$ in $(0, \infty)$. By induction, we choose integers $0 \leqslant N_{0}<\sigma(0) \leqslant N_{1}<\sigma(1) \leqslant \cdots$ such that the following three conditions are satisfied:

$$
\begin{array}{cl}
\left|\sum_{j=1}^{n}\left\langle y_{j}, f_{\sigma(m-1)}\right\rangle\right| \geqslant 1-\varepsilon_{m} \quad & \left(m \in \mathbb{N}, n \geqslant N_{m}\right) ; \\
\left\|\left(I_{V_{p}}-P_{k}\right) \sum_{j \in A} y_{j}\right\|_{V_{p}} \leqslant \varepsilon_{m} & \left(m \in \mathbb{N}, k \geqslant \sigma(m)-1, A \subseteq\left[1, N_{m}\right] \cap \mathbb{N}\right) ; \\
\sum_{j=N_{m}+1}^{\infty}\left|\left\langle y_{j}, f_{\sigma(k)}\right\rangle\right| \leqslant \frac{k \varepsilon_{m}}{m-1} & (m \geqslant 2,1 \leqslant k<m) .
\end{array}
$$

To start the induction, we observe that 6.8 -6.10 are vacuous for $m=0$, so we can simply take $N_{0}:=0$ and $\sigma(0):=1$. Now let $\ell \in \mathbb{N}$, and assume inductively that integers $0 \leqslant N_{0}<\sigma(0) \leqslant N_{1}<\sigma(1) \leqslant \cdots \leqslant N_{\ell-1}<\sigma(\ell-1)$ have been chosen such that 6.8 -6.10 are satisfied for each $m \leqslant \ell-1$. For $n \geqslant \sigma(\ell-1)$, our assumption 6.2 implies that

$$
1-\sum_{j=1}^{n}\left\langle y_{j}, f_{\sigma(\ell-1)}\right\rangle=\left\langle\chi_{[1, n]}-\sum_{j=1}^{n} y_{j}, f_{\sigma(\ell-1)}\right\rangle \rightarrow 0 \quad \text { as } \quad n \rightarrow \infty,
$$

so we can find $N_{\ell}^{\prime} \geqslant \sigma(\ell-1)$ such that 6.8 is satisfied for $m=\ell$ and each $n \geqslant N_{\ell}^{\prime}$. In 
the case where $\ell \geqslant 2, \ell-1$ applications of 6.7 yield a number $N_{\ell}^{\prime \prime} \in \mathbb{N}$ such that

$$
\sum_{j=N_{\ell}^{\prime \prime}+1}^{\infty}\left|\left\langle y_{j}, f_{\sigma(k)}\right\rangle\right| \leqslant \frac{k \varepsilon_{\ell}}{\ell-1} \quad(k \in\{1, \ldots, \ell-1\}) .
$$

Thus 6.8 and 6.10 will both be satisfied for each $m \leqslant \ell$ provided that we define

$$
N_{\ell}:= \begin{cases}N_{\ell}^{\prime} & \text { if } \ell=1 \\ \max \left\{N_{\ell}^{\prime}, N_{\ell}^{\prime \prime}\right\} & \text { otherwise. }\end{cases}
$$

Since there are only finitely many subsets of $\left[1, N_{\ell}\right] \cap \mathbb{N}$, and $\left\|\left(I_{V_{p}}-P_{k}\right) x\right\|_{V_{p}} \rightarrow 0$ as $k \rightarrow \infty$ for each $x \in V_{p}$ because $\left(e_{n}\right)_{n \in \mathbb{N}}$ is a basis for $V_{p}$, we can find $\sigma(\ell)>N_{\ell}$ such that 6.9 is satisfied for $m=\ell$, and hence the induction continues.

Now let $z_{m}:=\sum_{j=N_{m}+1}^{N_{m+1}} y_{j} \in V_{p}$ for each $m \in \mathbb{N}$; we then have

$$
\sum_{m=1}^{\infty}\left|\left\langle y_{m}, \xi_{\sigma}\right\rangle\right| \geqslant \sum_{m=1}^{\infty}\left|\sum_{j=N_{m}+1}^{N_{m+1}}\left\langle y_{j}, \xi_{\sigma}\right\rangle\right|=\sum_{m=1}^{\infty}\left|\left\langle z_{m}, \xi_{\sigma}\right\rangle\right| .
$$

Seeking a lower bound for the right-hand side of this inequality, we observe that

$$
\begin{aligned}
\left|\left\langle z_{m}, \xi_{\sigma}\right\rangle\right| & \geqslant\left|\left\langle P_{\sigma(m+1)-1} z_{m}, \xi_{\sigma}\right\rangle\right|-\left|\left\langle\left(I_{V_{p}}-P_{\sigma(m+1)-1}\right) z_{m}, \xi_{\sigma}\right\rangle\right| \\
& \geqslant\left|\sum_{k=1}^{\infty} \frac{(-1)^{k}}{k}\left\langle P_{\sigma(m+1)-1} z_{m}, f_{\sigma(k)}\right\rangle\right|-\left\|\xi_{\sigma}\right\|_{V_{p}^{\prime}}\left\|\left(I_{V_{p}}-P_{\sigma(m+1)-1}\right) z_{m}\right\|_{V_{p}} \\
& \geqslant\left|\sum_{k=1}^{m} \frac{(-1)^{k}}{k}\left\langle z_{m}, f_{\sigma(k)}\right\rangle\right|-\left\|\xi_{\sigma}\right\|_{V_{p}^{\prime}} \varepsilon_{m+1} \\
& \geqslant \frac{\left|\left\langle z_{m}, f_{\sigma(m)}\right\rangle\right|}{m}-\sum_{k=1}^{m-1} \frac{\left|\left\langle z_{m}, f_{\sigma(k)}\right\rangle\right|}{k}-\left\|\xi_{\sigma}\right\|_{V_{p}^{\prime}} \varepsilon_{m+1}
\end{aligned}
$$

for each $m \in \mathbb{N}$, where the penultimate inequality follows from $\sqrt{6.9}$ and the fact that

$$
P_{\sigma(m+1)-1}^{\prime} f_{\sigma(k)}= \begin{cases}f_{\sigma(k)} & \text { if } k \leqslant m \\ 0 & \text { otherwise. }\end{cases}
$$

If $m=1$, we ignore the second term on the right-hand side of 6.12 . Otherwise it has the following upper bound

$$
\sum_{k=1}^{m-1} \frac{\left|\left\langle z_{m}, f_{\sigma(k)}\right\rangle\right|}{k} \leqslant \sum_{k=1}^{m-1} \sum_{j=N_{m}+1}^{N_{m+1}} \frac{\left|\left\langle y_{j}, f_{\sigma(k)}\right\rangle\right|}{k} \leqslant \sum_{k=1}^{m-1} \frac{k \varepsilon_{m}}{k(m-1)}=\varepsilon_{m}
$$

by 6.10. We find a lower bound for the first term on the right-hand side of 6.12 by writing $z_{m}=\sum_{j=1}^{N_{m+1}} y_{j}-\sum_{j=1}^{N_{m}} y_{j}$ and using the fact that $P_{\sigma(m)-1}^{\prime} f_{\sigma(m)}=0$ :

$$
\left|\left\langle z_{m}, f_{\sigma(m)}\right\rangle\right|=\left|\sum_{j=1}^{N_{m+1}}\left\langle y_{j}, f_{\sigma(m)}\right\rangle-\left\langle\sum_{j=1}^{N_{m}} y_{j},\left(I_{V_{p}}-P_{\sigma(m)-1}\right)^{\prime} f_{\sigma(m)}\right\rangle\right|
$$




$$
\begin{aligned}
& \geqslant\left|\sum_{j=1}^{N_{m+1}}\left\langle y_{j}, f_{\sigma(m)}\right\rangle\right|-\left\|f_{\sigma(m)}\right\| V_{p}^{\prime}\left\|\left(I_{V_{p}}-P_{\sigma(m)-1}\right) \sum_{j=1}^{N_{m}} y_{j}\right\|_{V_{p}} \\
& \geqslant 1-\varepsilon_{m+1}-\varepsilon_{m}
\end{aligned}
$$

by (6.8), 6.9), and Lemma 4.3 i1. Substituting (6.13) and 6.14 into 6.12, we obtain

$$
\left|\left\langle z_{m}, \xi_{\sigma}\right\rangle\right| \geqslant \frac{1-\varepsilon_{m+1}-\varepsilon_{m}}{m}-\varepsilon_{m}-\left\|\xi_{\sigma}\right\|_{V_{p}^{\prime}} \varepsilon_{m+1} \geqslant \frac{1}{m}-\left(3+\left\|\xi_{\sigma}\right\|_{V_{p}^{\prime}}\right) \varepsilon_{m}
$$

Since the harmonic series diverges, whereas the series $\sum_{m=1}^{\infty} \varepsilon_{m}$ converges, we conclude that the series $\sum_{m=1}^{\infty}\left|\left\langle z_{m}, \xi_{\sigma}\right\rangle\right|$ diverges, and therefore, by (6.11), the same is true for the series $\sum_{m=1}^{\infty}\left|\left\langle y_{m}, \xi_{\sigma}\right\rangle\right|$, as desired.

Acknowledgements. We gratefully acknowledge the financial support from the EPSRC (Bird: EP/P500303/1, EP/P501482/1, and EP/P502656X/1; Laustsen: EP/F023537/1) that has enabled us to carry out the research which this paper is based upon.

Our main results were presented in two lectures at the $19^{\text {th }}$ International Conference on Banach Algebras held in Będlewo, Poland, $14^{\text {th }}-24^{\text {th }}$ July 2009. We would like to thank the organizers H. Garth Dales, Krzysztof Jarosz, Michał Jasiczak, and Andrzej Sołtysiak for their kind invitation to attend this conference and, in particular, for the opportunity to present our work there. The conference was financially supported by the Polish Academy of Sciences, the European Science Foundation under the ESF-EMSERCOM partnership, and the Faculty of Mathematics and Computer Science of the Adam Mickiewicz University in Poznań, a support which we gratefully acknowledge.

Last, but not least, we would like to thank András Zsák and Graham Jameson for many helpful discussions and comments.

\section{References}

[1] F. Albiac and N. J. Kalton, Topics in Banach Space Theory, Grad. Texts Math. 233, Springer-Verlag, 2006.

[2] D. E. Alspach and S. A. Argyros, Complexity of weakly null sequences, Dissertationes Math. 321 (1992).

[3] A. D. Andrew and W. L. Green, On James' quasi-reflexive space as a Banach algebra, Can. J. Math. 32 (1980), 1080-1101.

[4] A. Baernstein II, On reflexivity and summability, Studia Math. 42 (1972), 91-94.

[5] S. Banach and S. Saks, Sur la convergence forte dans les champs $L^{p}$, Studia Math. 2 (1930), 51-57.

[6] B. Beauzamy and J.-T. Lapresté, Modèles étalés des espaces de Banach, Publ. Dép. Math. (Lyon) 1983.

[7] C. Bessaga and A. Pełczyński, On bases and unconditional convergence of series in Banach spaces, Studia Math. 17 (1958), 151-164.

[8] C. Bessaga and A. Pełczyński, Banach spaces non-isomorphic to their Cartesian squares. I, Bull. Acad. Polon. Sci. Sér. Sci. Math. Astr. Phys. 8 (1960), 77-80.

[9] A. Bird, An amalgamation of the Banach spaces associated with James and Schreier, Part II: Banach-algebra structure, this volume, 35-43. 
[10] A. Bird, N. J. Laustsen, and A. Zsák, Some remarks on James-Schreier spaces, J. Math. Anal. Appl. 371 (2010), 609-613.

[11] A. Blanco, Weak amenability of $\mathscr{A}(E)$ and the geometry of E, J. London Math. Soc. 66 (2002), 721-740.

[12] A. Blanco and N. Grønbæk, Amenability of algebras of approximable operators, Israel J. Math. 171 (2009), 127-156.

[13] P. G. Casazza, James' quasi-reflexive space is primary, Israel J. Math. 26 (1977), 294-305.

[14] P. G. Casazza, B.-L. Lin, and R. H. Lohman, On James' quasi-reflexive Banach space, Proc. Amer. Math. Soc. 67 (1977), 265-271.

[15] P. G. Casazza and T. J. Shura, Tsirelson's Space, Springer Lecture Notes in Mathematics 1363, Springer-Verlag, 1989.

[16] N. Dunford and J. T. Schwartz, Linear Operators, Vol. I, Pure and Applied Mathematics 7, Interscience, 1958.

[17] I. S. Edelstein and B. S. Mityagin, Homotopy type of linear groups of two classes of Banach spaces, Functional Anal. Appl. 4 (1970), 221-231.

[18] H. Fetter and B. Gamboa de Buen, The James Forest, LMS Lecture Notes 236, Cambridge University Press, 1997.

[19] I. Gasparis and D. H. Leung, On the complemented subspaces of the Schreier spaces, Studia Math. 141 (2000), 273-300.

[20] D. P. Giesy and R. C. James, Uniformly non- $\ell_{1}$ and B-convex Banach spaces, Studia Math. 48 (1973), 61-69.

[21] R. Herman and R. Whitley, An example concerning reflexivity, Studia Math. 28 (1967), 289-294.

[22] R. C. James, Bases and reflexivity of Banach spaces, Ann. of Math. 52 (1950), 518-527.

[23] R. C. James, A non-reflexive Banach space isometric with its second conjugate space, Proc. Nat. Acad. Sci. 37 (1951), 174-177.

[24] N. J. Laustsen, K-theory for the Banach algebra of operators on James's quasi-reflexive Banach spaces, K-Theory 23 (2001), 115-127.

[25] N. J. Laustsen, Maximal ideals in the algebra of operators on certain Banach spaces, Proc. Edin. Math. Soc. 45 (2002), 523-546.

[26] N. J. Laustsen, Commutators of operators on Banach spaces, J. Operator Theory 48 (2002), 503-514.

[27] J. Lindenstrauss and L. Tzafriri, Classical Banach Spaces, Vol. I, Ergeb. Math. Grenzgeb. 92, Springer-Verlag, 1977.

[28] J. Lindenstrauss and L. Tzafriri, Classical Banach Spaces, Vol. II, Ergeb. Math. Grenzgeb. 97, Springer-Verlag, 1979.

[29] R. J. Loy and G. A. Willis, Continuity of derivations on $\mathscr{B}(E)$ for certain Banach spaces E, J. London Math. Soc. 40 (1989), 327-346.

[30] R. E. Megginson, An Introduction to Banach Space Theory, Grad. Texts Math. 183, Springer-Verlag, New York, 1998.

[31] E. W. Odell and H.-O. Tylli, Weakly compact approximation in Banach spaces, Trans. Amer. Math. Soc. 357 (2005), 1125-1159.

[32] A. Pełczyński, A connection between weakly unconditional convergence and weakly completeness of Banach spaces, Bull. Acad. Polon. Sci. Sér. Sci. Math. Astr. Phys. 6 (1958), $251-253$. 
[33] G. Pisier, The dual $J^{*}$ of the James space has cotype 2 and the Gordon-Lewis property, Math. Proc. Camb. Phil. Soc. 103 (1988), 323-331.

[34] J. Schreier, Ein Gegenbeispiel zur Theorie der schwachen Konvergenz, Studia Math. 2 (1930), 58-62.

[35] G. A. Willis, Compressible operators and the continuity of homomorphisms from algebras of operators, Studia Math. 115 (1995), 251-259. 